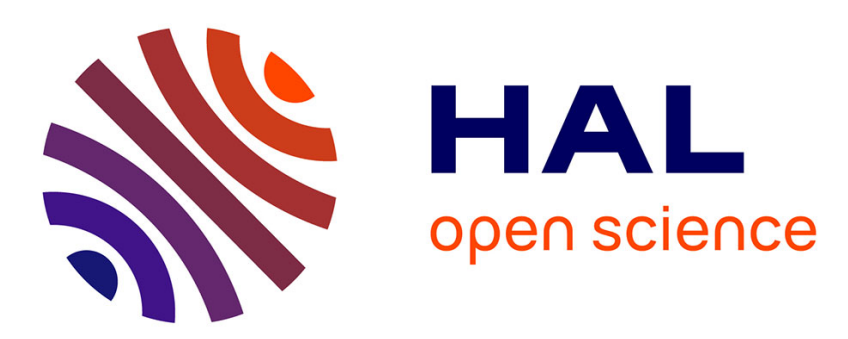

\title{
Des poissons, des mots et des signes : les signes monastiques des noms de poissons au XIe siècle
}

Catherine Jacquemard, Marie-Agnès Lucas-Avenel

\section{To cite this version:}

Catherine Jacquemard, Marie-Agnès Lucas-Avenel. Des poissons, des mots et des signes: les signes monastiques des noms de poissons au XIe siècle. Annales de Normandie, 2012, 62 (2), pp.139-174. 10.3917/annor.622.0139 . hal-00783809

\section{HAL Id: hal-00783809 \\ https://hal.science/hal-00783809}

Submitted on 6 Jul 2020

HAL is a multi-disciplinary open access archive for the deposit and dissemination of scientific research documents, whether they are published or not. The documents may come from teaching and research institutions in France or abroad, or from public or private research centers.
L'archive ouverte pluridisciplinaire HAL, est destinée au dépôt et à la diffusion de documents scientifiques de niveau recherche, publiés ou non, émanant des établissements d'enseignement et de recherche français ou étrangers, des laboratoires publics ou privés. 


\title{
Des poissons, des mots et des signes : les signes monastiques des noms de poissons au xle siècle
}

\author{
Catherine JACQuemard* et Marie-Agnès LUCAS-AVEnEL**
}

Entre le mont et Tumbeleine

Cort tost la meir par mié l'areine.

Plenté i a de granz saumons,

De lamprees, d'autres peissons,

Quer l'en i prent et muls et bars,

Bons esturgons et granz sabars,

Torboz, plaïz, congres, harens,

Porpeis, graspeis, quant en est tens,

E tanz menuz peissons de meir

Que nes vos sei demiés nommeir.

\begin{abstract}
Entre le mont et Tombelaine, la mer sillonne rapidement le sable. Il y a là en abondance des grands saumons, des lamproies et d'autres poissons : on $\mathrm{y}$ prend des mulets, des bars, de bons esturgeons et de grands sabars, des turbots, des plies, des congres, des harengs, des marsouins, des baleines quand c'est l'époque, et tant de petits poissons de mer que je ne saurais vous en nommer la moitié

Guillaume de Saint-Pair, Roman du Mont Saint-

Michel, v. 465-474, éd. C. Bougy ${ }^{1}$ )
\end{abstract}

ORSQUE le moine Guillaume de Saint-Pair, au milieu du XII ${ }^{\mathrm{e}}$ siècle, a entrepris de raconter la fondation du Mont Saint-Michel par l'évêque Aubert, il a ressenti la nécessité d'interrompre son récit pour vanter la diversité et la richesse de la faune aquatique de la baie. Ces quelques vers nous rappellent à quel point la pêche, encadrée par une réglementation complexe de droits de pêche et de redevances multiples, est alors une activité essentielle. Pour des raisons aussi bien économiques que religieuses (de nombreux interdits pèsent sur la consommation de la viande sur presqu'un tiers de l'année), qu'il s'agisse du poisson des pauvres, le hareng, ou des mets habituels des tables des puissants, comme les lamproies ou les saumons, les poissons tiennent une place considérable dans l'alimentation des hommes du Moyen Âge, et c'était tout particulièrement vrai pour les communautés monastiques. Si certaines espèces peuvent se conserver facilement une fois séchées ou salées et supporter ainsi un long transport, la plupart du temps ce sont les poissons frais, les espèces locales, qui sont consommées. On ne s'étonnera donc pas que Guillaume de Saint-Pair, dans son abbaye au péril de la mer, exprime son intérêt pour les espèces maritimes qui devaient arriver à la cuisine et au réfectoire de son monastère, mais les poissons d'eau douce ont fait l'objet d'une attention aussi marquée dans les

\footnotetext{
* Professeur de latin, CRAHAM, UMR 6273, université de Caen Basse-normandie, F 14032 Caen.

** Maître de Conférences en latin, CRAHAM, UMR 6273, université de Caen Basse-normandie, F 14032 Caen

1 Guillaume de Saint-Pair, Le roman du Mont Saint-Michel, C. Bougy (éd.), Presses universitaires de Caen - Avranches : Scriptorial, 2009.
} 
abbayes situées plus loin des rivages. C'est ainsi que les listes de signes rédigées au XI ${ }^{\mathrm{e}}$ siècle dans les abbayes de Cluny, Fleury ou Hirsau accordent une place non négligeable aux noms des espèces aquatiques familières aux moines.

Pas moins de vingt espèces de " poissons » étaient consommées dans l'abbaye bénédictine d'Hirsau à la fin du XI ${ }^{\mathrm{e}}$ siècle d'après ce qu'enseigne le catalogue de signes composé par l'abbé Guillaume ${ }^{2}$. La langue des signes, pratiquée dans l'abbaye-mère de Cluny probablement dès sa fondation ${ }^{3}$, était le seul mode de communication permis dans la cuisine ou le réfectoire, où la règle du silence devait être rigoureusement respectée, comme dans l'église et le dortoir ${ }^{4}$ :

"Chacun se doit d'apprendre diligemment les signes, grâce auxquels on parle en quelque manière sans prononcer un mot, car, quand on s'est uni à une communauté monastique, on n'a que très rarement la permission de parler. Et voici quels sont les lieux, dans le cloître, où, selon la tradition de nos Pères et la règle fixée par eux, on doit observer un silence absolu : l'église, le dortoir, le réfectoire et la cuisine régulière. $»^{5}$

Les catalogues clunisiens rassemblent 118 mots que les moines sont susceptibles d'avoir besoin d'exprimer sans contrevenir à la règle ${ }^{6}$. Ils furent enseignés

2 Le coutumier de Guillaume a été composé avant la mort de l'abbé en 1091 sur le modèle de celui d'Ulrich de Zell dont on parlera ci-après (cf. note 7). Il a été édité récemment par P. ENGELBERT, Willehelmi abbatis Constitutiones Hirsaugienses, vol. 1, Siegburg, F. Schmitt (Corpus consuetudinum monasticarum ; 15), 2010 ; pour les signes des poissons, cf. p. 199-203 et ci-après.

3 Odon avait certainement été initié à la langue des signes quand il entra comme novice à Baumeles-moines, avant de devenir abbé de Cluny (925-942), comme l'indique son biographe, JEAN DE SALERnE, Vita Odonis, PL 133, col. 57Ab : Nam, quoties necessarias ad exposcendum res instabant, toties diversa in invicem fiebant ad perficiendum signa, quas puto grammatici digitorum et oculorum notas vocare voluerunt. Adeo nempe inter eos excreverat ordo iste, ut puto si sine officio linguae essent, ad omnia necessaria significanda sufficere possent signa ipsa, « en effet, chaque fois qu'ils étaient contraints de demander quelque chose, ils utilisaient divers signes qu'ils s'adressaient l'un à l'autre pour y parvenir : à mon avis les grammairiens les eussent appelés signes faits avec les doigts et les yeux. En effet, cette règle était si bien établie chez eux que, d'après moi, s'ils avaient perdu l'usage de la langue, les signes à eux seuls auraient pu suffire à exprimer toutes les choses nécessaires ». Pour une histoire de la langue des signes dans les abbayes clunisiennes depuis la fondation de l'abbaye de Cluny en 910 jusqu'à la fin du XII ${ }^{\mathrm{e}}$ siècle, on pourra consulter notamment S.G. BRuCE, Silence and Sign Language in Medieval Monasticism, The Cluniac Tradition c. 900-1200, Cambridge, Cambridge University Press, 2007. La communication par signes ou gestes est même bien antérieure à Cluny ou même à la Règle de Benoît : cf. L. Gougaud, « Le langage des silencieux », Revue Mabillon, 19, 1929, p. 93-100 (réimpr. dans Monastic Sign Languages, J. Umiker-Sebeok et T. A. Sebeok (dir.), Berlin, New York, Amsterdam, Mouton de Gruyter, 1987, p. 5-12).

4 Les règles du silence sont exposées dans le chapitre qui précède le catalogue des signes de Cluny (symbolisé par C), intitulé De silentio in quibus officinis sit tenendum, « Sur le silence et les lieux où il faut le garder ». Elles furent reprises à Fleury $(\mathrm{F})$ et Hirsau $(\mathrm{H})$ avec quelques modifications, mais les auteurs s'accordent sur la nécessité absolue de respecter le silence dans ces lieux.

5 Hirsau, ch. IV (P. Engelbert (éd.), op. cit., p. 194-195) : Opus quoque habet, ut signa diligenter addiscat, quibus tacens quodammodo loquatur, quia postquam adunatus fuerit ad conuentum, licet ei rarissime loqui. Et tales officine in claustro, in quibus est traditum a patribus nostris et praefixum, ut perpetuum silentium teneatur, id est, ecclesia, dormitorium, refectorium, et coquina regularis. L'extrait a été emprunté par Guillaume d'Hirsau aux coutumiers de Cluny (cf. ci-dessous).

6 Un plus grand nombre de signes cependant étaient en usage, comme en témoigne la définition même de certains signes faisant appel à des signes non transcrits. C'est le cas par exemple du signe du saumon composé du signe général des poissons et de celui de l'orgueil (cf. ci après); or, le signe de l'orgueil ne figure pas dans le catalogue de Cluny. D'après S. G. Bruce, Silence and Sign Language..., op. cit., p. 71, 
et transcrits à la fin du XI ${ }^{\mathrm{e}}$ siècle par Bernard et Ulrich de Cluny, qui les insérèrent dans leur coutumier7. Adoptés aussi à Fleury-sur-Loire, peut-être dès la venue d'Odon dans l'abbaye en 938, les signes furent rassemblés dans un catalogue au sein du coutumier composé ou copié en $1087^{8}$ : les 154 signes qu'il contient ont été rédigés selon le modèle clunisien. Guillaume d'Hirsau fit de même, mais porta le nombre de mots à environ $359^{9}$. La pratique des signes se répandit encore aux $\mathrm{XII}^{\mathrm{e}}$ et XIII ${ }^{\mathrm{e}}$ siècles dans les abbayes d'Europe occidentale, comme en attestent son adoption par les Cisterciens et les nombreuses traductions en langues vernaculaires ${ }^{10}$.

Chaque entrée du catalogue associe un lexème et un signe chargé de dénoter le lexème : le signe correspond toujours à un geste de la main. S'y ajoute parfois une explication sur le choix du geste. Cette syntaxe, invariable au sein du catalogue clunisien, n'a pas été modifiée par les catalogues rédigés postérieurement. On y trouve ainsi des listes de mots - surtout des substantifs désignant des objets ou des individus, très peu de verbes -, regroupés sous diverses catégories : alimentation (pains, poissons, fromages, légumes), vêtements, objets liturgiques, livres, personnes ayant une charge spécifique, prières et offices etc. En cela, ils donnent des informations très utiles sur la vie quotidienne des moines

ces lacunes dans le catalogue prouvent que la composition de celui-ci avait une visée didactique, et que les novices n'étaient instruits que des signes jugés indispensables.

7 Pour Bernard de Cluny, cf. Ordo cluniacensis sive consuetudines, M. Herrgott (éd.), in Vetus disciplina monastica, Paris, C. Osmont, 1726, p. 136-364 (réimpr. Siegburg, F. Schmitt, 1999) : le catalogue des signes, «De notitia signorum », occupe le chapitre XVII, p. 169-173. Ulrich DE ZelL, Consuetudines cluniacenses, PL 149, col. 635-778 : le catalogue des signes, « De signis loquendi », occupe le chapitre IV du livre II, col. 703-705. On se réfèrera cependant aux textes des catalogues édités par W. JARECKI, Signa loquendi, Die cluniacensischen Signa-Listen eingeleitet und herausgegeben, V. Koerner, Baden-Baden (Saecula spiritalia ; 4), 1981, p. 121-142. Les spécialistes ne sont pas tous d'accord sur les dates exactes de composition des coutumiers de Bernard de Cluny et d'Ulrich. Retenons ici celles qui ont été établies par J. Wollash, «Zur Verschriftlichung der klösterlichen Lebensgewohnheiten unter Abt Hugo von Cluny », Frühmittelalterliche Studien, 27, 1993, p. 317-349, dont les conclusions ont été reprises par S. G. BRUCE, Silence and Sign Language..., op. cit., p. 9. Celui de Bernard daterait de 1078 ou juste après et celui d'Ulrich de 1079-1084.

8 Le copiste du XIV e siècle qui a transmis le catalogue (ms Orléans, Bibl. mun. 2293) a recopié le colophon de son modèle, qui porte la date du 7 décembre 1087 : cf. S. G. Bruce, Silence and Sign Language..., op. cit., p. 105 et n. 26 ; A. DAVRIL, « Le langage par signes chez les moines, Un catalogue de signes de l'abbaye de Fleury », dans Sous la règle de saint Benoît, Structures monastiques et sociétés en France du Moyen Âge à l'époque moderne, [actes du colloque tenu à l']Abbaye bénédictine Sainte-Marie de Paris, 23-25 octobre 1980, Genève, Droz (Centre de recherches d'histoire et de philologie de la IVe section de l'École Pratique des Hautes Études, série V, Hautes Études médiévales et modernes ; 47), 1982, p. 55. Le catalogue a été publié par ibid., p. 55-70, et W. JARECKI, Signa loquendi, op. cit., p. 250-275.

9 Le catalogue des signes de Guillaume d'Hirsau édité par W. JARECKI, «Signa loquendi », op. cit., p. 163-230, fait apparaître 359 signes, mais le nombre varie selon les manuscrits : certains signes, adoptés par P. ENGELBERT (éd.), op. cit., ont été rejetés dans l'apparat critique par W. JARECKI : nous nous contenterons de mentionner les variantes qui concernent les poissons (cf. ci-après).

10 Cf. S. G. Bruce, Silence and Sign Language..., op. cit., ch. 5, « Continuity and criticism », p. 143170 ; R. A. Barakat, Cistercian Sign Languages : a study in non-verbal communication, Cistercian Studies Series, 7, Kalamazoo, Michigan : réimpr. dans Monastic Sign Languages, op. cit., p. 67-322. On pourra aussi se reporter à G. VAN RINJBERK, Le langage par signes chez les moines, Amsterdam, NorthHolland Publishing Company, 1953, qui donne, dans l'ordre alphabétique, le texte latin de tous les signes employés dans les abbayes depuis le $\mathrm{XI}^{\mathrm{e}}$ jusqu'au XIX ${ }^{\mathrm{e}}$ siècle. 
dans les abbayes bénédictines, d'autant que la comparaison de ceux-ci et l'étude de leurs différences témoignent de la manière dont les abbayes ont adapté le modèle clunisien à leurs propres usages ${ }^{11}$. Cependant, comme le choix du geste n'a pas été arbitraire, mais tient compte des connaissances expérimentales des acteurs de la situation de communication, ces catalogues constituent aussi des témoins intéressants de ce que l'association du lexème et de son signe révèle du signifié ainsi dénoté.

Dans cet article, nous nous intéresserons aux poissons et animaux aquatiques, qui constituent l'objet d'étude du programme «Ichtya » mené au Centre Michel de Boüard (CRAHAM) : nos recherches sur la transmission du savoir ichtyologique au Moyen Âge ont porté sur l'étude et l'édition d'œuvres encyclopédiques, dont les informations reposent essentiellement sur un savoir livresque transmis depuis l'Antiquité. C'est pourquoi, nous profitons de l'occasion que nous donnent les Mélanges en l'honneur de notre collègue Catherine Bougy, pour examiner ces signes linguistiques si particuliers figurant dans les catalogues de Cluny, Fleury et Hirsau et désignant les poissons. Après avoir donné une traduction placée en regard des textes édités par nos prédécesseurs, nous tenterons de justifier l'identification de chaque poisson indiquée dans la traduction et de dégager les critères retenus pour figurer les poissons et nous nous demanderons si leurs choix reflètent une assez bonne connaissance des animaux cuisinés et consommés pour qu'on puisse y voir une source du savoir ichtyologique.

\section{I. - TEXTE ET TRADUCTION}

Les trois catalogues de la fin $\mathrm{du} \mathrm{XI}{ }^{\mathrm{e}}$ siècle retenus pour cet article - ceux de Cluny, Hirsau et Fleury - présentent entre eux des écarts qui ne s'expliquent pas seulement par des accidents dans la transmission textuelle d'un même modèle, mais qui témoignent aussi de variations induites par des approches différentes (usages, connaissances, dénominations, signes) des espèces animales citées.

La traduction que nous proposons a été réalisée à partir du texte établi par W. Jarecki pour Cluny et Fleury ${ }^{12}$, tandis que nous avons préféré, pour Hirsau, le texte revu par Engelbert. Il ne pouvait être question de reproduire l'apparat critique de chacun de ces éditeurs ; cependant, nous indiquons d'une note les variantes portant sur le nom d'un poisson ou affectant la compréhension du

11 Voir, pour Fleury-sur-Loire, S. G. BRUCE, Silence and Sign Language..., op. cit., p. 105-106 : il mentionne à titre d'exemple l'ajout du signe du hareng; pour Hirsau, voir ibid. p. 122-123 : il se réfère aussi notamment au nombre plus important qu'ailleurs de signes désignant les poissons.

12 W. JARECKI, Signa loquendi, op. cit. 
geste $^{13}$. Afin de faciliter la lecture comparative des noms de poissons dans les trois catalogues où ils ne sont pas ordonnés semblablement, nous avons opté pour l'ordre alphabétique, mettant en regard chaque fragment de texte - numéroté comme dans l'édition de W. Jarecki, y compris pour Hirsau - et sa traduction. On trouvera cependant en annexe à cet article un tableau comparatif, qui respecte l'ordre choisi par chaque auteur.

$<$ Cluny $=$ Hirsau $=$ Fleury $>$

$\mathrm{De}^{14}$ signis loquendi

De ipsis ${ }^{15}$ autem signis $u^{16}{ }^{16}$ aliqua ponam verbi ${ }^{17}$

gratia et primum que ad victum pertinent. [...]

\section{Piscis « le poisson»}

$<$ Cluny $8=$ Hirsau 11 $>$

Pro signo generali piscium cum manu simula caude piscis in aqua commotionem.

$<$ Fleury 12>

Pro generali signo piscium cum tota manu simila caudam piscis facere et move sicut piscis caudam movet in aqua.

\section{$\mathrm{Al}(1) \mathrm{ec}$ « le hareng »}

$<$ Hirsau 22>

Pro signo allecis praemisso generali signum salis adde.

$<$ Fleury 13>

Pro signo alecis, generali signo premisso, hoc adde ut cum pollice et indice obstruas oculos sicut dicitur quod tu nichil vides plus quam alec.
La langue des signes

Voici ce que, par la grâce des mots, j’établirai au sujet de ces signes, en commençant par ceux qui concernent la nourriture.

[...]

Pour le signe général des poissons, imite avec la main le mouvement de la queue du poisson dans l'eau.

Pour le signe général des poissons, imite avec toute ta main le mouvement de la queue du poisson et bouge-la comme le poisson bouge sa queue dans l'eau.

Pour le signe du hareng, après avoir fait le signe général <des poissons>, ajoute celui du sel.

Pour le signe du hareng, après avoir fait le signe général < des poissons>, ajoute celuici : bouche-toi les yeux avec le pouce et l'index, comme pour dire que tu ne vois pas mieux que le hareng.

13 Le ms Ky omet les signes suivants : piscis, sturio, salmo, lahso, murena siue lampreda, sepiae, lucius, asco.

14 de signis loquendi non hab. F.

15 ipsis $C H$ : hiis $F$.

16 ut non hab. $H$.

17 verbi gratia non hab. $H$. 


\section{Anguilla « l'anguille »}

$<$ Cluny 10 = Hirsau $19=$ Fleury 15>

10 Pro signo anguille cumclude utramque manum, quasi qui ita tenet et premit anguillam.

\section{Asco « l'ombre»}

$<$ Hirsau 25 apparat>

Pro signo piscis qui asco ${ }^{19}$ appellatur praemisso generali hoc adde, ut summitatem indicis super pollicis ungulam in ante prosilire facias, ac si simules auferentem ipsius squamas ${ }^{20}$.

\section{Piscis assus « le poisson grillé »}

$<$ Hirsau 25>

Pro signo piscis assi contra indicem eminentem aliquantulum flatus emitte, postea cum eodem sibique proximo cratem simula, disiunctos eos et inuersos tenens.

\section{Barbo « le barbeau »}

<Hirsau 20>

Pro signo piscis qui barbo uocatur ${ }^{21}$ praemisso generali hoc adde, ut cum duobus digitis simules componentem grenones.

\section{Brashima « la brème »}

< Hirsau 23>

Pro signo brahsime ${ }^{22}$ praemisso generali hoc adde, ut manum extendas, latitudinem ipsius simulans.
10 Pour le signe de l'anguille, ferme tes deux mains, comme quelqu'un qui tient et serre une anguille de cette manière ${ }^{18}$.

Pour le signe du poisson qu'on appelle l'ombre commun, après avoir fait le signe général < des poissons>, ajoute celui-ci : projette vers l'avant la pointe de ton index sur l'ongle de ton pouce, comme pour imiter quelqu'un qui écaille le poisson.

Pour le signe du poisson grillé, souffle légèrement sur le haut de ton index, puis, avec ce doigt et celui qui en est le plus proche, fais comme une grille, en les tenant séparés et placés en sens inverse.

Pour le signe du poisson qu'on appelle le barbeau, après avoir fait le signe général < des poissons $>$, ajoute celui-ci : avec deux doigts, imite quelqu'un qui arrange ses moustaches.

Pour le signe de la brème, après avoir fait le signe général < des poissons>, ajoute celuici : étend la main pour figurer la largeur de l'animal.

18 Pour ce poisson les catalogues de Petrus Boherius (11) et de Saint-Victor (25), édités dans W. Jarecki, Signa loquendi, op. cit., précisent qu'il est "glissant» (respectivement : labilis et labentem).

19 Certains manuscrits portent des variantes orthographiques au nom asco, d'autres donnent l'équivalence du nom latin traditionnel, timallus : voir l'apparat critique établi par P. ENGELBERT (éd.), op. cit., p. 202 : asco] hasco $V H$ ascho $B$ Mü $M n$ id est timallus s. v. al. m. add. A timallus s.v. add. Ad timallus id est asco $L z$.

20 pro-squamas] om. Ky.

21 piscis - uocatur] barbonis $B$.

22 brahsime] brahesimae $M \ddot{u} M n$ brasime $M c$ prasimae $H$. 


\section{Cancer $«$ le crabe $»^{23}$}

$<$ Hirsau 26>

Pro signo cancri praemisso generali forpicis signum adde ${ }^{24}$.

\section{Carpho « la carpe »}

$<$ Hirsau 17>

Pro signo piscis qui uulgari nomine carpho ${ }^{25}$ dicitur praemisso [generali] signo pugnum fronti imprime, quod est signum porci.
Pour le signe du crabe, après avoir fait le signe général <des poissons>, ajoute celui des ciseaux.
Pour le signe du poisson qu'on appelle communément la carpe, après avoir fait le signe [général] <des poissons >, place ton poing sur le front, ce qui est le signe du porc.

\section{Lampreda « La lamproie »; Murena siue lampreda « la murène ou la lam- proie"}

$<$ Cluny 11>

Pro signo lamprede in maxilla cum digito simula punctos tres vel quatuor propter ipsos punctos quos lampreda subtus oculos habet.

Pour le signe de la lamproie, feins de faire avec un doigt trois ou quatre points sur ta machoire, à cause des points que la lamproie a au-dessous des yeux.

$<$ Hirsau15>

Pro signo murene siue lamprede ${ }^{26}$ in maxilla cum digito simula punctos tres uel quatuor.

Pour le signe de la murène ou de la lamproie, avec un doigt fais comme si tu marquais trois ou quatre points sur ta mâchoire.

$<$ Fleury 16>

Pro signo murene ${ }^{27}$ vel lampride in maxilla simila cum indice tres punctos vel quatuor propter illos punctos quos subtus oculos habet.

\section{Pour le signe de la murène ou de la} lamproie, feins de faire avec un doigt trois ou quatre points sur ta machoire, à cause des points qu'elle a au-dessous des yeux.

\section{Lasho « le saumon »}

$<$ Hirsau 14>

Pro signo lahsonis ${ }^{28}$ idem quod et salmonis addito signo similitudinis.

Pour le signe du labson, c'est le même que celui du saumon, en y ajoutant le signe de la ressemblance.

23 Sur les difficultés posées par la traduction de cancer, voir commentaire ci-après.

24 pro - adde] om. $M c$.

25 carpho] charpho $M n$ karpho $K y$ charpo $K$.

26 siue lamprede] id est $V m g$. $a d d$. lampfrid $A$ lamfredae $L z$ al. lampredae $m g$. $H$.

27 murene] musene F Davril.

28 lahsonis] lassonis $H$. 


\section{Lucius « Le brochet »}

\section{$<$ Cluny 13>}

Pro signo lucii iterum generali signo premisso piscium hoc adde, $\mathrm{ut}^{29}$ cum manu signum facias celeritatis, quia lucius celerius quam alius piscis natat.

\section{$<$ Hirsau 16> \\ Pro signo lucii ${ }^{30}$ praemisso [generali] signo hoc adde, ut manum dextram naribus superponas, summitatibus digitorum sursum eminentibus.}

\section{$<$ Fleury 18>}

Pro signo lucii, generali signo premisso, hoc adde ut iungas summitates pollicis et indicis et addas oculo, sicut si aspiceres per aliquod foramen.
Pour le signe du brochet, de même après avoir fait le signe général des poissons, ajoute celui-ci : fais avec la main le signe de la rapidité, car le brochet nage plus vite que tout autre poisson.

Pour le signe du brochet, après avoir fait le signe [général] <des poissons>, ajoute celui-ci : place ta main droite sur ton nez, en levant la pointe de tes doigts vers le haut.

Pour le signe du brochet, après avoir fait le signe général <des poissons>, ajoute celui-ci : joins la pointe de ton pouce à celle de ton index et approche-les de ton œil, comme si tu regardais par un trou.

\section{Pisces minuti « les très petits poissons $»^{31}$}

$<$ Hirsau 24 apparat>

Pro signo piscium minutorum generali praemisso signum paruae rei adde.

\section{Minuua « la chevesne?»}

$<$ Hirsau 24 apparat>

Pro signo minuuae ${ }^{32}$ generali praemisso manum dexteram extentam per medium pollicis et indicis alterius manus quasi ferrum limando trahe, quod etiam signum cuiusque rei est quam in frequenti usu habemus.
Pour le signe des très petits poissons, après avoir fait le signe général <des poissons>, ajoute celui de ce qui est petit.

Pour le signe de la minuna [la chevesne ?], après avoir fait le signe général < des poissons>, étends ta main droite et tire-là au milieu du pouce et de l'index de l'autre main comme si tu aiguisais un couteau, car c'est aussi le signe de ce que nous utilisons fréquemment.

29 ut - natat] ut cum manu superficiem nasi conplanes quia hic piscis longum rostrum habere videtur L14 g14 (d'après W. Jarecki, Signa loquendi, op. cit., p. 123).

30 lucii] s.v. add. hachid $A A d$ id est hachid $L z$.

31 Ce signe et le suivant n'apparaissent que dans les manuscrits V H K Mc. Les deux fragments placés successivement dans le texte de Guillaume édité par P. Engelbert, op. cit., p. 203 - mais dans l'ordre inverse à celui adopté ici pour les besoins de l'ordre alphabétique -, sont rejetés comme une addition par W. JARECKI, Signa loquendi, op. cit., p. 167.

32 Outre le fait que ce nom de poisson n'apparaît que chez Guillaume et seulement dans certains manuscrits, la graphie de son nom a été diversement interprétée par les copistes : minuuae] muniwae $K$ muniuue $M c$ munuae $H$. 


\section{Muletus « le mulet»}

$<$ Fleury 20>

Pro signo muleti, hoc adde ut digitum surgas sicut si faceres modum saltandi eo quod cito $^{33}$ vertitur et saltat in aqua.

Pour le signe du mulet, <après avoir fait le signe général des poissons>, ajoute celui-ci : relève ton doigt pour faire comme un saut, parce que ce poisson se tourne rapidement et bondit dans l'eau.

\section{Ruppa « la lote»}

$<$ Hirsau 24>

Pro signo ruppe praemisso generali saxi signum adde.

Pour le signe de la lote, après avoir fait le signe général <des poissons>, ajoute celui du rocher.

\section{Salmo « le saumon »; salmo vel sturio « le saumon ou l'esturgeon »}

$<$ Cluny 12>

Pro signo salmonis vel sturionis premisso generali signo piscium hoc adde, ut pugnum erecto pollice subponas mento, quo superbia significatur, quia superbi maxime et divites tales pisces solent habere.

\section{$<$ Hirsau 13> \\ Pro signo salmonis praemisso signo hoc adde, ut duos digitos circa oculum ponas.}

$<$ Fleury 17>

Pro signo salmonis, generali signo premisso, hoc adde ut pugnum excepto ${ }^{34}$ pollice supponas mento quia superbia significatur, eo quod superbi et divites tales pisces solent habere.
Pour le signe du saumon ou de l'esturgeon, après avoir fait le signe général des poissons, ajoute celui-ci : place ton poing avec le pouce levé sous le menton - c'est le signe de l'orgueil-, car ce sont surtout les orgueilleux et les riches qui consomment habituellement ces espèces de poissons.

Pour le signe du saumon, après avoir fait le signe < général des poissons>, ajoute celuici : place deux doigts autour de ton œil.

Pour le signe du saumon, après avoir fait le signe général <des poissons>, ajoute celuici : place sous le menton ton poing avec le pouce sorti $\mathrm{i}^{35}$, parce que c'est le signe de l'orgueil et que ce sont les orgueilleux et les riches qui consomment habituellement ces espèces de poissons.

33 cito] sitto F ex Jarecki scito Davril.

34 excepto] erecto corr. Davril ex $B U$ (BU sont les sigles utilisés pour désigner Bernard et Ulrich).

35 Nous traduisons ici le texte de la tradition manuscrite, mais la correction d'A. DAVRIL, « Le langage par signes chez les moines ", op. cit., est sans doute juste (cf. note précédente). 


\section{Sepia « la seiche»}

$<$ Cluny 9 = Fleury 14>

Pro signo sepiarum ${ }^{36}$ divide omnes digitos $a b$ invicem et ita eos commove, quia et sepie ${ }^{37}$ ita multiplices esse videntur.

$<$ Hirsau 15 apparat $>$

$\mathrm{Pro}^{38}$ signo sepiarum disiunge omnes digitos $\mathrm{ab}$ inuicem et ita eos commoue.

\section{Sturio « l'esturgeon »}

$<$ Hirsau 12>

Pro signo sturionis praemisso [generali] signo hoc adde, ut summitatem pollicis mento supponas.

\section{Truta « la truite »}

$<$ Cluny 14 = Fleury 19>

Pro signo trute ${ }^{39}$ hoc adde, ut digitum de supercilio $^{40}$ ad supercilium trahas propter ligaturas ${ }^{41}$ que hoc in loco habentur a feminis, et quia truta ${ }^{42}$ femineo genere pronuntiatur.

\section{$<$ Hirsau 18>}

Pro signo truite ${ }^{43}$ praemisso generali hoc adde, ut digitum de supercilio ad supercilium trahas, quod ${ }^{44}$ est signum feminae, propter ${ }^{45}$ ligaturas quae in tali loco habentur a feminis, $\mathrm{et}^{46}$ quia truita semper feminino genere pronunciatur.
Pour le signe des seiches, sépare tous tes doigts les uns des autres, et, ainsi disposés, bouge-les, car les seiches passent pour avoir de multiples bras.

Pour le signe des seiches, sépare tous tes doigts les uns des autres, et, ainsi disposés, bouge-les.

Pour le signe de l'esturgeon, après avoir fait le signe [général] <des poissons>, ajoute celui-ci : place la pointe de ton pouce sous ton menton.

Pour le signe de la truite, <après avoir fait le signe général des poissons $>$, ajoute celui-ci : fais passer ton doigt d'un sourcil à l'autre à cause des bandes que les femmes portent en cet endroit, car la truite est du genre féminin.

Pour le signe de la truite, après avoir fait le signe général <des poissons>, ajoute celui-ci : fais passer ton doigt d'un sourcil à l'autre - c'est le signe de la femme -, à cause des bandes que les femmes portent en cet endroit, car la truite est toujours du genre féminin.

36 sepiarum $C$ : sepium $F$.

37 sepie $C$ : sepa $F$.

38 pro - commoue] hab. VH KMc om. cett. Selon W. Jarecki, Signa loquendi, op. cit., p. 166, ce fragment est une addition.

39 Précisons pour Cluny : trute Jarecki] truite Herrgott.

40 Précisons pour Cluny: supercilio... supercilium] supercilicio... supercilium $c$ (d'après W. JARECKI, Signa loquendi, op. cit., p. 124). Pour Fleury : supercilio... supercilium] superlicio... superlicium F Davril. La forme superlicium du ms de Fleury résulte probablement d'une métathèse pour supercilium : le sens d'« habit de chanoine régulier ", recensé par NIERMEYER et DU CANGE, pour superlicium d'après superpellicium semble bien loin du témoignage donné par Cluny et Hirsau.

41 Précisons pour Fleury : ligaturas] lignaturas F Davril. Là encore, il nous semble que W. JARECKI, Signa loquendi, op. cit., a raison d'écarter lignatura, « redevance en bois».

42 truta Jarecki] truita Herrgott.

43 truite] tructae $V$ uorihen s.v. add. A Ad vorhen s.v. add. Lz id est forehen add. Ky.

44 quod-feminae] om. $K M c$.

45 propter - feminis] hab. VH KMc om. cett.

46 et quia-pronunciatur] hab. KMc om. cett. 


\section{II. - COMMENTAIRES ${ }^{47}$}

Les trois lexiques des signes relatifs aux poissons établis à la fin $\mathrm{du} \mathrm{XI} \mathrm{XI}^{\mathrm{e}}$ siècle dans les abbayes bénédictines de Fleury, Cluny et Hirsau s'ouvrent sur le même signe générique qui rend compte du mot piscis et mime le déplacement d'un poisson dans l'eau : cum manu simula caude piscis in aqua commotionem / cum tota manu simila caudam piscis facere et move sicut piscis caudam movet in aqua. Toutefois les listes incluent dans le même ensemble un crustacé, cancer, "le crabe ", et un mollusque, sepia, "la seiche »; il faut donc comprendre piscis au sens très large d'animal aquatique qu'il recouvre dans l'Antiquité comme au Moyen Âge.

On ne relève que quatre signes semblables qui soient associés aux mêmes vocables par chacune des trois listes : anguilla, l'anguille, lampreda, la lamproie, sepia, la seiche, truita / truta, la truite. Les trois lexiques ont encore en commun, mais traduits par des signes spécifiques différents, les espèces suivantes : salmo, le saumon, les listes de Fleury et Cluny recourant au même signe évocateur, mais Hirsau choisissant une autre caractérisation, et lucius, le brochet, avec une détermination différente dans chacun des trois catalogues. Les lexiques de Fleury et d'Hirsau nomment encore en commun le poisson al(l)ec, le hareng, mais avec deux signes différents. Le catalogue de Fleury est seul à mentionner le muletus, le mulet, tandis que celui d'Hirsau, globalement beaucoup plus riche que les listes restreintes de Cluny et de Fleury, possède 11 entrées qui lui sont propres : asco, l'ombre, piscis assus, le poisson grillé, barbo, le barbeau, brashima, la brème, cancer, le «crabe ", carpho, la carpe, lasho, le saumon, pisces minuti, les très petits poissons, minuиa, la chevesne (?), ruppa, la lote, sturio, l'esturgeon.

47 Nous avons utilisé pour nos recherches les instruments offerts par les bases de données : Acta Sanctorum Database (mise en ligne par ProQuest LLC des Acta sanctorum de la Société des Bollandistes, désormais cités AASS) ; Brepolis (corpus textuels des Library of Latin texts, séries A et B, et des Monumenta Germaniae Historica, désormais désignés sous les abréviations LLT et eMGH ; Database of Latin dictionnaries, désormais DLD, qui donne accès aux dictionnaires suivants : E. Forcellini, G. FurLAnetto, Fr. Corradini, J. Perin, Lexicon Totius Latinitatis, 1940 ; E. Forcellini, J. Perin, Lexicon Totius Latinitatis. Onomasticon (auctore J. Perin), Patavii, Typis seminarii, 1940, 6 vol. ; Ch.T Lewis, Ch. Short, A Latin Dictionary, Oxford, Clarendon Press, 1879; A. BLAIse, Dictionnaire latin-français des auteurs chrétiens, Turnhout, Brepols, 1967 ; A. BlaIse, Lexicon latinitatis medii aevi, Turnhout, Brepols, 1975 (Dictionnaire latin-français des auteurs du Moyen Âge) ; Du CANGE, Glossarium ad scriptores mediae et infimae latinitatis conditum a Carolo du Fresne Domino du Cange, editio nova a L. Favre, 10 vol., Niort 18831887 [réimpr. Graz, Akademische Druck- und Verlagsanstalt, 1954] ; Firmini Verris Dictionarius, [Dictionnaire latin-français de Firmin Le Ver], B. Merrilees, W. Edwards (éd.), Turnhout, Brepols, 1994 ; Anonymi Montepessulanensis Dictionarius, A. Grondeux (éd.), Turnhout, Brepols, 1998 ; GuILlaume LE Talleur, Dictionarius familiaris et compendiosus, W. Edwards, B. MerrileEs (éd.), Turnhout, Brepols, 2002 ; A. Souter, A glossary of Later Latin to 600 A.D, Oxford, Clarendon Press, 1949 [réimpr. 1957]. Nous avons aussi consulté les notices étymologiques d'A. ERnout et A. Meillet, Dictionnaire étymologique de la langue latine, Paris, Klincksieck, $1967^{4}$ (abrégé par la suite en Ernout-Meillet), de P. Chantraine, Dictionnaire étymologique de la langue grecque, Paris, Klincksieck, 1990, et pour la langue française, les notices du Centre national de ressources textuelles et lexicales mises en ligne par l'ATILF (CNRTL) et celles du Dictionnaire historique de la langue française, sous la direction d'A. REY, Dictionnaires Le Robert, Paris, $1998^{2}$ (cité infra A. REY). 
Les trois listes reposent sur le même principe de formation du lexique : le signe désignant chaque espèce particulière de poisson est un signe double, avec, pour premier élément, le signe générique traduisant piscis et, pour second élément, un signe spécifique évocateur de l'espèce qu'on veut désigner. Nous n'avons relevé qu'un seul cas de combinaison de trois éléments distincts : pour évoquer le lasho, le saumon, le catalogue d'Hirsau associe au signe générique du poisson ceux du saumon et de la ressemblance, à moins qu'il ne fasse l'économie du signe générique. Le second élément peut être un signe préexistant, doté d'une signification propre et susceptible de fonctionner de façon autonome, la formation du double signe étant alors similaire à la formation d'un mot composé. Il peut aussi correspondre à un élément qualificatif réservé à ce seul emploi, sans existence autonome, dans une sorte de nomenclature binaire. Les deux procédés semblent avoir été également utilisés. Neuf doubles signes sont explicitement définis comme composés de deux signes autonomes : al(l)ec, le poisson-sel ou le hareng (Hirsau 22) ; cancer, le poisson-pinces / ciseaux ou le «crabe " (Hirsau 26) ; carpho, le poisson-porc ou la carpe (Hirsau 17) ; lucius, le poisson-vitesse ou le brochet (Cluny 13); pisces minuti, les poissons-petitesse (Hirsau 24) ; minuua, le poisson-« usage fréquent » ou la chevesne (?) (Hirsau 24) ; ruppa, le poisson-roche ou la lote (Hirsau 24) ; salmo, le poisson-orgueil ou le saumon (Cluny 12, Fleury 17) ; truta / truita, le poisson-femme ou la truite (Cluny 14, Fleury 19, Hirsau 18). Le deuxième type de formation puise dans un répertoire de signes inspirés par la physiologie, le comportement ou l'usage du poisson. On peut ainsi relever des signes qualificatifs tirés d'une particularité physique : anguilla, l'anguille, caractérisée par sa peau visqueuse ; barbo, le barbeau, par les barbillons de sa lèvre supérieure ; brashima, la brême, par sa largeur ; lampreda, la lamproie, par ses ouvertures branchiales ; sepia, la seiche, par ses bras. D'autres signes jouent sur le comportement de l'animal : ainsi muletus, le mulet, est évoqué par ses bonds ; et d'autres sont basés sur l'usage : le signe de l'asco, l'ombre, mime le fait d'écailler le poisson, et celui des poissons grillés, pisces assi, la cuisson sur un gril. Quelques signes sont plus énigmatiques et reposent sur des similitudes dont les codes implicites nous échappent en grande partie aujourd'hui ; nous y reviendrons en reprenant l'étude des listes signe par signe.

Si, à l'intérieur d'une même liste, il n'y a pas de réel cas d'ambiguïté ou d'homonymie, en revanche, un élément spécifique a pu servir à caractériser, selon les communautés monastiques, des espèces différentes. Ainsi le signe de l'orgueil a été retenu par les catalogues de Cluny et de Fleury comme qualificatif du salmo, le saumon, tandis qu'un geste similaire, mais sans explication étiologique, caractérise le sturio, l'esturgeon dans la liste d'Hirsau. De même pour nommer le lucius, le brochet, il est demandé à Cluny et Fleury d'entourer l'œil des doigts, tandis que ce geste, sans être accompagné d'une quelconque justification, est réservé au salmo, le saumon, dans la liste d'Hirsau. 
Nous examinons maintenant les entrées des trois listes en regardant, dans un premier temps, les signes propres à Fleury et à Hirsau et, dans un second temps, les signes communs aux trois monastères; cependant nous ne traiterons pas deux entrées du catalogue d'Hirsau, piscis assus, le poisson grillé, et minuti pisces, les petits poissons, qui ne sont pas des noms d'animaux à proprement parler.

\section{A. - Les signes propres à Fleury et à Hirsau}

\section{1. - Signe commun à Fleury et à Hirsau}

Les catalogues de Fleury et d'Hirsau ont en commun l'entrée allec, mais dont le signe diffère d'un catalogue à l'autre.

\section{Al(l)ec (Fleury 13, Hirsau 22), le hareng}

Le terme (b)allec / (b)allex est bien attesté dans l'Antiquité, où il désignait soit une sorte de marinade, soit le poisson qui entrait dans sa composition ${ }^{48}$. Pline (nat. 31, 44) nomme (b)allex une sauce à base de menuise décomposée et comparable au garum, mais distingue le condiment du poisson qu'il

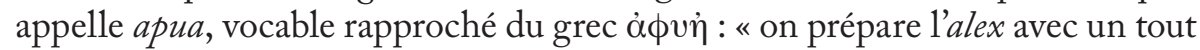

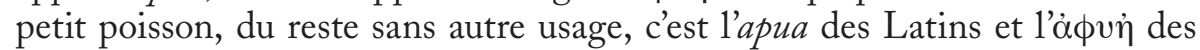
Grecs ", il précise par ailleurs que ses contemporains ne se satisfont plus de la recette de base, mais qu'ils préparent une infinité d'allex à partir d'huîtres, de hérissons de mer, d'orties marines, de homards, de foies de surmulet...; en revanche, Isidore de Séville (orig. 12, 6, 39) donne le même nom au poisson et à la préparation dans laquelle il entre « l'allec est un tout petit poisson bon pour la saumure liquide, d'où son nom ». Il existait aussi un diminutif allicula qui renvoie chez Columelle à un petit poisson de rivière, peut- être le vairon (Phoxinus laevis Linné, 1758). Le terme allec est passé dans les langues romanes avec des représentants pour la sardine, l'anchois et l'alose ${ }^{49}$. Dans la littérature latine médiévale, le terme semble s'être spécialisé dans la désignation du hareng (Clupea harengus Linné, 1758), ainsi l'allec est présenté par Vincent de Beauvais, Thomas de Cantimpré ou Albert le Grand, comme un poisson spécifique de la mer du Nord et de la Manche ${ }^{50}$ qu'ils distinguent de l'alose

48 Voir De SAInT-Denis, Le vocabulaire des animaux marins..., op. cit., p. 45.

49 Voir le commentaire de J. André, dans Isidore de Séville, Étymologies, livre XII, J. André (éd.), Paris, Les Belles Lettres, 1986, 204, n. 384 ; H. Walter et P. AvenAs, La fabuleuse histoire du nom des poissons, Paris, Robert Laffont, 2011, p. 135, notent que allec est à l'origine de it. alice pour anchois.

50 Vincent de Beauvais, Speculum naturale, Douai, 1624 [reprint Graz, 1964], 17, 30 ; Albert le Grand, De animalibus, 24, 2 (7) ; Thomas de CANTIMPRÉ, Liber de natura rerum, Editio princeps secundum codices manuscriptos, H. Boese (éd.), Berlin-New-York, Walter de Gruyter, 1973, 7, 5. En revanche, la Chronique de Mathias de Nuwenburg (SS rer. Germ. NS 4) évoque des «allecia » pêchés dans un lac près de Cologne qui ne peuvent pas être des harengs... 
nommée par son appellation vernaculaire verith/verich. Le Summarium Heinrici ${ }^{51}$ confirme cette spécialisation de sens de allec en fournissant la glose allec berinc (qu'on rapprochera de all. heering, häring, la forme passant aussi dans le calque latin aringus sous différentes graphies). Hildegarde de Bingen (Physica 5,22 et 18$)^{52}$, de même, distingue les poissons allec et meysisch, ce dernier étant la grande alose. Le hareng, la sardine ou l'alose sont des clupéidés très proches d'apparence, mais contrairement à l'alose et à la sardine, le hareng est un poisson de l'Atlantique et des mers du Nord qui ne se rencontre pas plus bas que le golfe de Gascogne. La pêche et le commerce du hareng (qui se conserve longtemps une fois salé) donnent lieu à une importante activité (bien attestée dans les sources médiévales). Il entre dans l'alimentation courante, en particulier des plus pauvres, et son abondance est considérée comme un don de la bienveillance divine ${ }^{53}$.

Si le signe, proposé par la liste d'Hirsau, qui imite le sel dans une allusion au mode de conservation d'un poisson qui était consommé plus souvent salé que frais, surtout à l'intérieur des terres, s'explique fort bien, en revanche le signe proposé par le catalogue de Fleury reste énigmatique. La description de l'allec chez les encyclopédistes médiévaux nous suggère des explications possibles, mais dont aucune n'emporte, à notre avis, l'adhésion. Ainsi on peut lire, par exemple, chez Thomas de Cantimpré (Liber de natura rerum 7, 5) : «La nuit, ses yeux brillent dans la mer comme des lanternes, mais ils perdent ce pouvoir à la mort du poisson. Ils se rassemblent en bancs autour des lumières partout où ils en voient briller en mer au-dessus des eaux. C'est une astuce dont on se sert pour les attirer dans les filets au moment voulu, comme si un don de Dieu les avait réservés à la pêche pour la consommation de l'homme. L'hiver,

51 Les dix livres du Summarium Heinrici reprennent et actualisent les Étymologies d'Isidore de Séville en les complétant d'extraits de Priscien, Cassiodore ou Bède ; le texte est accompagné de gloses précisant les équivalents vernaculaires d'un certain nombre de termes spécialisés. Le Summarium Heinrici, dont on ne connaît que le prénom de l'auteur grâce au poème liminaire acrostiche, aurait été composé à, ou près de, Worms entre les années 1007-1022. Nous avons consulté le texte du Summarium Heinrici dans l'édition de R. Hildebrand, Summarium Heinrici, Berlin, de Gruyter, 1974, ainsi que dans l'étude de W. Wegstein, Studien zum "Summarium Heinrici » : Die Darmstädter Handschrift 6, Tübingen, Max Niemeyer, 1985. Toutes les références au Summarium Heinrici sont empruntées à Summarium Heinrici 3, 16.

52 Hildegarde de Bingen (1098-1179) a composé la Physica aussi connue sous le nom Subtilitatum diversarum naturarum creaturarum libri novem au milieu du XII ${ }^{\mathrm{e}}$ siècle. Nous avons consulté le texte du livre 5, le Liber de piscibus dans le volume de la PL 197 c. 1269-1286 et dans la récente édition critique de R. Reiner Hildebrandt et T. Gloning : Hildegard von Bingen, Physica Liber subtilitatum diversarum naturarum creaturarum: Textkritische Ausgabe, Berlin - New York, de Gruyter, 2010, p. 259-285. Selon l'analyse de R. Hildebrandt et T. Gloning, le texte original de la Physica aurait comporté, pour le vocabulaire spécialisé, à la fois les termes allemands et leurs équivalents latins (les gloses latines pourraient avoir été le fait de Volmar, le scribe et confesseur d'Hildegarde), l'original ayant été perturbé par les aléas de la tradition manuscrite. Nous avons aussi utilisé la traduction de P. MonAt, Hildegarde de Bingen, Le livre des Subtilités des Crétures Divines, Grenoble, Jérôme Million, 1989. Sur le vocabulaire employé par Hildegarde, voir L. Moulinier, "La faune germanique médiévale : une brève histoire de noms », in Milieux naturels, espaces sociaux. Études offertes à Robert Delort, F. Morenzoni, É. Mornet (éd.), 1997, p. 193-208.

53 Par exemple, Alain Des Iles, De planctu naturae, cap. 2 : Illic allec, piscis generalissimus, ampla sui communitate pauperum solabatur ieiunia; ou encore THOMAS DE CANTIMPRÉ, op. cit. 7,5 : ad retia dictis temporibus alliciuntur, quasi parati ad capiendum divino munere in usus hominum deducuntur. 
ils se réfugient dans les eaux profondes au large de la Germanie tout le temps nécessaire ». Le hareng avait donc la réputation d'avoir les yeux brillants dans la mer, tandis qu'il perdait ce pouvoir dès qu'il était mort. Est-ce la raison pour laquelle les moines attirent l'attention sur les yeux privés de vie du hareng une fois qu'il est mort? Ou veulent-ils faire allusion au fait que les bancs serrés de harengs (éblouis par les lanternes) se précipitent dans les filets des pêcheurs comme s'ils ne les voyaient pas?

\section{2. - Signe propre à Fleury}

La liste de Fleury n'a qu'une seule entrée qu'on ne retrouve ni dans la liste de Cluny ni dans celle d'Hirsau.

Muletus (Fleury 20) : le mulet.

Il existe de nombreuses espèces de muges ou mulets qui sont des poissons vifs ; ils émigrent en été dans les estuaires et les lagunes et s'avancent souvent assez loin en remontant les cours d'eau. Il est donc difficile de caractériser plus le muletus de la liste de Fleury, mais il pourrait ici s'agir du mulet sauteur (Mugil saliens ou Liza saliens Risso, 1810), le signe retenu insistant sur les bonds effectués par l'animal. Le mot muletus pour désigner le mulet apparaît tardivement en latin (avec les hésitations graphiques muletus, mulletus, mulettus enregistrées par le dictionnaire Du Cange). C'est un diminutif dérivé de mulus, qui désignait dans la langue classique, un « mulet », mais l'hybride de l'âne et de la jument et non le poisson. Un paronyme écrit avec une géminée, mullus, servait bien à nommer en latin un poisson, mais il s'agissait du rouget, sous les deux espèces du rouget-barbet de vase (Mullus barbatus Linné, 1758), commun en Méditerranée, et du rouget-barbet de roche ou surmulet (Mullus surmuletus Linné, 1758), qu'on rencontre sur les rivages de l'océan Atlantique, de la Manche et de la mer du Nord. En revanche, c'est le terme mugil que la langue classique réservait aux mulets, et plus particulièrement à l'espèce réputée pour son agilité, le mulet sauteur, qu'on peut reconnaître dans les descriptions de Pline (Plin. nat. 9, 54) ou d'Isidore de Séville (Isid. orig. 12, 6, 26) ${ }^{54}$. Les encyclopédistes médiévaux conservent la nomenclature du latin classique et ne font pas mention d'un poisson nommé muletu ${ }^{55}$, mais ils s'appuient essentiellement sur une tradition livresque remontant à Isidore ou Pline ; cependant Albert le Grand distingue mullus, au sens de surmulet, et mulus, qui serait le mulet à

54 Sur la distinction opérée en latin classique entre mugil et mullus, voir E. DE SAINT-Denis, Le vocabulaire des animaux marins en latin classique, Paris, Klincksieck, 1947, p. 66-69.

55 Les bases de données Brepolis et AASS fournissent peu d'attestations de mul(l)et(t)us. On relève ainsi dans AASS, Apr. I, Dies 3, une occurrence dans la Vita de Richard de Chichester, rédigée par RALF BROCKING peu après la canonisation de Richard en 1262 (lib. 1, cap 7) : educentes vero rete in terram, invenerunt pisces quatuor admodum pulchros, qui vulgo muletti dicuntur, cujusmodi in aqua illa capi non solebant; sunt enim marini potius quam fluviales. 
grosses lèvres (Mugil chelo Cuvier, 1829 ou Chelon labrosus Risso, 1827) ${ }^{56}$. Si on considère la postérité des deux termes dans les langues romanes, on constate que mugil pour désigner le mulet s'est maintenu tout au long de la latinité et qu'il a produit des représentants romans bien attestés fr. muge, it. muggine, mugello, et muge, esp. múgil, ptg. Mugem. Il ne semble pas en être de même pour mul(l)us, qui, au sens de rouget-barbet, ne paraît pas avoir connu une grande fortune dans les langues romanes ${ }^{57}$. L'ancien français mul (ca 1170, Guillaume de Saint-Pair, Roman du Mont Saint-Michel, 469), dont est dérivé mulet (fin du XII ${ }^{\mathrm{e}}$ siècle, Moniage Guillaume, II, 1026), est issu du latin mul(l)us, mais il semble avoir eu le même sens que son diminutif mul(l)et ${ }^{58}$. Quoi qu'il en soit, le signe choisi par les moines de Fleury confirme qu'il faut reconnaître un mulet dans le poisson nommé muletus, avec un glissement de sens par rapport au latin classique. Le latin médiéval muletus, attesté tardivement, correspond peut-être à la latinisation du terme vernaculaire mulet, plutôt qu'à une dérivation directe à partir du latin mulus. L'emploi de muletus aux dépens de mugil (ou des noms vernaculaires fr. mulet, angl. mullet aux dépens des descendants de mugil) pourrait être un particularisme régional propre à la France et à l'Angleterre, c'est ainsi du moins qu'il a été compris par les philologues des $\mathrm{XVI}^{\mathrm{e}}$ et XVII ${ }^{\mathrm{e}}$ siècles comme l'humaniste italien Polidoro Vergilio ${ }^{59}$, Gesner ${ }^{60}$, ou encore Henschenius et Papebrochius dans leur édition de la Vita de Richard de Chichester ${ }^{61} \ldots$

56 Nous adoptons les identifications proposées dans les édition et traduction consultées : ALBERTUS Magnus, De animalibus libri XXVI nach der Cölner Urschrift, H. Stadler (éd.), Münster, Aschendorff, 1916-1920 ; Albertus Magnus, On Animals : A Medieval Summa Zoologica, K. F. Kitchell, I. M. ResNICK (trad.), Baltimore, Johns Hopkins University Press, 1999.

57 Une première recherche sur le site Fishbase ne nous a permis de relever que l'espagnol moll. Le Nomenclator aquatilium animantium de GESNER (Nomenclator aquatilium animantium. Icones animalium aquatilium... plus quam D C C., cum nomenclaturis singulorum latinis, graecis, italicis, hispanicis, gallicis, germanicis, anglicis... per certos ordines digestae... Nominum confirmandorum causa descriptiones quorundam et alia quaedam, praesertim in magno nostro de aquatilibus volumine non tradita, adduntur... per Conradum Gesnerum..., Tiguri, excudebat C. Froschoverus, 1560, p. 39) ne recense déjà plus qu'une désignation vernaculaire du rouget qui puisse venir du latin mullus : l'ancien. français mö̈l relevé par RondELET (et il donne la forme comme largement concurrencée par de nombreuses autres appellations dans le domaine français).

58 Voir sur ce point les notices étymologiques du CNRTL et d'A. REY, s.v. mulet. L'homonymie des désignations du quadrupède et du poisson, favorisée par les fluctuations dans la notation de la géminée, aura probablement entraîné un glissement de sens du rouget au mulet pour le poisson. La notice du CNRTL consacrée au fr. mulet note que l'aspect du poisson évoque la lourdeur des formes [on peut aussi ajouter la couleur] du quadrupède : or cette ressemblance vaut peut-être pour le mulet, mais certes pas pour le rouget-barbet.

59 Polidoro Vergilio, Anglica Historia (lib. 1, cap. 17) : piscium item omnis generis copia redundat, verum multorum nomina non eadem atque Latina sunt. Capo vocatur gornardus, asellus wytingus, mugiles sive cephali muletti...

60 Nomenclator aquatilium animantium, op. cit., p. 69 : scio Anglos a Gallis mutuatos mugilem appellant a Mullet.

61 AASS, Apr. I, Dies 3, Vita S. Richardi Episcopi per Fr. Radulphum lib. 1, cap. 7, en note de l'occurrence muletti, les éditeurs du volume (Henschenius et Papebrochius) ont précisé : Muletti non Anglis tantum, sed etiam Francis dicuntur: Latinis Mugiles. 


\section{3. - Signes propres à Hirsau}

Seules deux entrées propres au catalogue d'Hirsau sont d'origine latine : cancer, le «crabe » et barbo, le barbeau.

Cancer (Hirsau 26) : « le crabe »

Le terme cancer désigne en latin classique de façon générique les crustacés marins aussi bien que ceux d'eau douce (le cancer fluviatilis correspondant le plus souvent à l'écrevisse, Astacus astacus Linné, 1758). Il recouvre des espèces aujourd'hui rangées dans l'ordre des décapodes, qu'il s'agisse de natantia, « ceux qui nagent » (les crevettes), ou, parmi les reptantia, " ceux qui marchent ou rampent ", des macroures (les homards, les langoustines, les langoustes, les cigales de mer et, en eau douce, les écrevisses), des anomoures (en particulier, le pagure ou bernard-l'ermite) et des brachyoures (les crabes proprement dits, avec leurs plus grands représentants : le tourteau commun et l'araignée de mer). Le mot a conservé sa polysémie en latin médiéval, si bien qu'il faut ici le prendre au sens large de crustacé, d'autant plus que le geste de mimer des pinces ou des ciseaux peut aussi bien convenir aux crabes proprement dits qu'aux homards ou aux écrevisses. Toutefois, dans le contexte d'une liste établie pour l'usage d'une abbaye aussi éloignée de la mer qu'Hirsau, il se pourrait que cancer ait plus souvent désigné l'écrevisse d'eau douce que les crustacés marins.

\section{Barbo (Hirsau 20), le barbeau}

À la différence de cancer qui est attesté depuis la latinité classique, le mot barbo n'apparaît que dans les sources médiévales, alors qu'on rencontre la forme barbus dès le IV ${ }^{\mathrm{e}}$ siècle, chez Ausone (Mos. 94) ${ }^{62}$, pour désigner un poisson de rivière. C'est cette appellation barbus, ou son diminutif barbellus, qui est à l'origine des noms vernaculaires du barbeau (Barbus barbus Linné, 1758), un poisson des eaux claires et rapides dont la chair est agréable et qui se reconnaît en particulier à ses barbillons. Gesner ${ }^{63}$ relève ainsi les noms barbio, barbo (Italie), bárbo, báruo (Espagne), barbeau, barbet (France ${ }^{64}$ et Barb, Barben, Barbel (Allemagne). Dans le contexte du catalogue d'Hirsau, le mot barbo doit s'interpréter plutôt comme la forme vernaculaire barbo attestée en haut allemand (et issue de latin barbus) que comme un nom directement transmis du latin. Hildegarde de Bingen (Physica 5, 10) traite en effet la forme barbo comme les autres termes vernaculaires qu'elle emploie, sans la décliner (avec un ablatif et

62 Ausone : Ausonio, La Mosella, A. Marsili (éd.), Turin, Loescher editore, 1957.

63 Nomenclator aquatilium animantium, op. cit., p. 307.

64 Le français barbeau est attesté à la fin du XII siècle sous la forme barbiaux, pluriel de barbel: ainsi, vers 1178, dans Le roman de Renart, E. MarTin (éd.), Strasbourg, K.J. Trübner, Paris, E. Leroux, 1882-1887, branche III, v. 393 : «Les anguilles et les barbiaus Et autres poissons bons et biaus ». Voir notice du CNRTL s.v. barbeau. 
un nominatif identiques barbo), et le Summarium Heinrici donne baruo comme une appellation germanique qu'il glose par le latin silurus ${ }^{65}$. Le signe retenu, qui sert aussi pour le chat, catta, avec une allusion aux moustaches, grenones ${ }^{66}$, est l'illustration de la particularité physique la plus remarquable du barbeau, mais il est vrai qu'il aurait pu servir à nommer bien d'autres poissons : loches, goujons, carpes, lotes et, bien sûr, silures... Toutefois, le rapprochement avec les formes vernaculaires issues de barbus qui convergent toutes vers la désignation du barbeau nous fait privilégier pour barbo le sens de barbeau à celui de silure indiqué par le Summarium Heinrici ${ }^{67}$.

Les entrées asco (Hirsau 25), l'ombre, brashima, la brème (Hirsau 23), carpho (Hirsau 17), la carpe, lasho (Hirsau 14), le saumon, minuua (Hirsau 24), la chevesne (?), ruppa (Hirsau 24), la lote, sturio (Hirsau 12), l'esturgeon, correspondent toutes à des termes d'origine germanique.

\section{Asco (Hirsau 25), l'ombre}

Le nom asco est à rapprocher de l'allemand Asche, Äsche qui désigne l'ombre commun (Thymallus thymallus Linné, 1758), un salmonidé des eaux claires et froides, aujourd'hui menacé de disparition dans nos cours d'eau, mais alors très répandu ${ }^{68}$. Certains témoins manuscrits des Constitutiones Hirsaugienses glosent asco par le nom latin thymallus : asco id est timallus; et le Summarium Heinrici fournit la même équivalence : timallus asch [leçon donnée par W. Wegstein] ascho [leçon donnée par R. Hildebrandt]). Le nom latin de l'ombre commun thymallus, qu'on rencontre par exemple chez Ambroise (hex. 5, 2, 6) ou Isidore de Séville (orig. 12, 6, 29), est un dérivé de thymus / thymum, le thym,

65 Le silure possède aussi des barbillons caractéristiques. Les éditions consultées de la Physica d'HiLDEgarde DE Bingen donnent aussi la glose Barbo i. silurus. En Hongrie, c'est également le latin barbo qui a été choisi pour traduire le nom vernaculaire du silure Hartsa : voir, par exemple, J.B. Grossinger, Universa Historia physica regni Hungariae secundum tria regna naturae digesta, t. III, Posonii et Comaromii, P. Weber, 1794, passim.

66 Signe Hirsau 357 dans W. Jarecki, Signa loquendi, op. cit., p. 231 : pro signo catte, duobus digitis componentem grenones simula. Le mot grano / greno, grenonis peut aussi désigner les nattes de cheveux (comme grani mentionné par Isid. orig. 19, 23, 7), ou la barbe, les favoris. Voir Geoffroy de Viterbe, Pantheon (a.o.m. - 1186 [1191]), G. WAITZ (éd.), MGH SS 22, Particula XXIII, cap. 29 : Dum tenet Ottonem, barbam trahit atque granonem, / Guttur et ora premens, gravior feritate leone; voir aussi L. D’ACHÉRY, Veterum aliquot scriptorum qui in Galliae Bibliothecis, maxime Benedictinorum, latuerant, Spicilegium Tomus Secundus... opera et studio D. Lucae d'Acherii, Parisiis, apud Carolum Savreux, 1657, p. 435 (ca a. 1135): Barbati et prolixos habentes granos.

67 Il est, par ailleurs, difficile d'interpréter dans quel sens a joué la glose du Summarium Heinrici: le glossateur connaissait-il la signification exacte des deux termes barbo et silurus ? A-t-il réinterprété a posteriori l'ancien mot latin silurus comme désignant le même animal que le vernaculaire barbo?

68 Hildegarde de Bingen consacre une notice à un poisson nommé De ascha (Physica 5, 20) : L. Moulinier, «La faune germanique médiévale », op. cit., p. 196, considère qu'il s'agit de l'ombre commun, mais P. Monat, Hildegarde de Bingen, op. cit., p. 110, traduit ascha par l'omble (nom qui désigne communément un autre poisson : Salvelinus alpinus Linné, 1758). 
et s'explique par le parfum que dégage la chair de l'ombre (le poisson perd son goût caractéristique quelques heures après sa mort). Le signe retenu (le geste de mimer l'écaillage du poisson) s'explique peut-être par la disposition remarquable de ses écailles alignées en rangées longitudinales bien distinctes les unes des autres.

\section{Brashima (Hirsau 23), la brème}

Le terme brashima remonte à un étymon francique *brabsima qu'on peut déduire du néerlandais brasem, du danois brasen, du francais brème ${ }^{69}$, de l'allemand Brachsen (m. b. all. brassem, avec chute du $b$ devant $s$, a. h. all. brabsema, brabsima), qui sont les appellations de la brème commune (Abramis brama Linné, 1758). C'est un poisson très répandu dans les eaux à courant lent, résistant (facile à transporter) et de chair agréable ${ }^{70}$. Le mot remonterait à une racine germanique brebwan signifiant «briller ». Le signe choisi qui attire l'attention sur la largeur du poisson, latitudo, pourrait s'expliquer par l'aspect morphologique de la brème qui est dotée d'un corps haut et comprimé latéralement.

\section{Carpho (Hirsau 17), la carpe}

Le mot est à considérer comme la transcription d'une forme vernaculaire, qu'on rapprochera du nom de la carpe en allemand Karpfen, plutôt que comme une variante du bas latin carpa. Le latin carpa (le latin médiéval connaît aussi les formes carpo, carpio, carpana, relevées par Du Cange), est, par ailleurs, luimême un emprunt à une langue de l'Europe orientale par l'intermédiaire du got. karpa. L'appellation carpa a pénétré tardivement dans la Romania, et sa plus ancienne attestation se trouve chez Cassiodore, qui évoque la carpe, au début $\mathrm{du} \mathrm{VI}^{\mathrm{e}}$ siècle, comme un poisson du Danube : Destinet carpam Danuvius (Cass. var. 12, 4, 1) ${ }^{71}$. La carpe commune (Cyprinus carpio carpio Linné, 1758) est, en effet, restée jusqu'à une date récente confinée au bassin des moyen et bas Danube. Ce n'est qu'entre le $\mathrm{VII}^{\mathrm{e}}$ et le $\mathrm{XI}^{\mathrm{e}}$ siècle, mais surtout au $\mathrm{XI}^{\mathrm{e}}$ siècle, que la carpe est passée du bassin du Danube aux affluents du moyen Rhin. Jusqu'à la

69 L'ancien français braisme est attesté au XII siècle (Moniage Guillaume, 1027 : braismes [var. brasmes, braisnes, braimes, braines, bremes] et moruaus salés); on rencontre av. 1250 bresmes (Huon de Cambrai, Regrets N.D., 70, 9). Voir notice de CNRTL s.v. brème.

70 Nos recherches sur la base Brepolis ne nous ont pas permis de retrouver d'autres occurrences de brabsima sous les différentes graphies qu'auraient pu prendre le mot prabsima / brasima / brebsima... Peut-être s'agit-il du poisson brenna ou bregma évoqué brièvement par les encyclopédistes médiévaux : Alexandre Neckam, De naturis rerum libri duo with the poem of the same author, De laudibus divinae sapientiae, Thomas Wright (éd.), Londres, Longman, 1863, 33 ; Vincent de Beauvais, Speculum naturale, op. cit., 17, 33 ; Marcus d'Orvieto, Liber de moralitatibus (Urbevetanus Marcus, Marci de Urbe Veteri, O.F.M. Liber de moralitatibus), G.J. ETZKORn (éd.), St. Bonaventure, N. Y., St. Bonaventure University, $2005,4,80 \ldots$

71 Voir notice du CNRTL s.v. carpe. 
fin du XI ${ }^{\mathrm{e}}$ siècle, rien n'indique qu'elle ait été domestiquée, les sources littéraires sur la carpe restent rares (les Constitutiones Hirsaugienses constituent de ce point de vue un précieux témoignage). Au début du XII ${ }^{\mathrm{e}}$ siècle encore, Alexandre Neckam ne mentionne pas la carpe parmi les animaux aquatiques, ce qui laisse à penser qu'elle n'était pas encore parvenue dans le nord de la France. Mais du $\mathrm{XII}^{\mathrm{e}}$ siècle au début du XIV ${ }^{\mathrm{e}}$, la carpe gagne les cours de la Meuse et du Rhin et, de là, le Bassin parisien et la Bourgogne. De nombreux textes parlent alors de la carpe, qu'il s'agisse de réglementations sur la pêche et l'élevage, de recueils encyclopédiques ou de livres de recettes... Pour la première fois, on trouve des allusions précises à l'élevage de la carpe. Au cours d'une troisième étape, au milieu du XIV ${ }^{\mathrm{e}}$ siècle, la carpe aurait enfin atteint le sud-ouest de la France et se serait répandue, au nord, jusqu'en Angleterre et en Scandinavie ; on ne la trouverait en Italie qu'à la fin du Moyen Âge $\mathrm{e}^{72}$.

Le signe pour désigner la carpe, le poisson-porc, peut s'expliquer par l'allure générale, compacte et ramassée, de la carpe et du sanglier ou du porc. Mais le signe a pu être aussi inspiré par une certaine ressemblance de la bouche protactile de la carpe, qui lui permet de fouiller la vase, avec le groin du sanglier et du porc.

\section{Labso (Hirsau 14), le saumon}

Le terme labso (ici donné au génitif labsonis) est la forme latinisée et déclinable d'une appellation vernaculaire du saumon dans le domaine germanique (ahd et mhd lahs, nhd Lachs). Le signe retenu joue sur la similitude du poisson nommé labso avec le saumon, salmo, mais chacun des deux animaux est doté d'une entrée propre couplée avec un signe spécifique. Le Summarium Heinrici donne l'équivalence : esox labs (avec la variante labse). Ainsi les témoignages des Constitutiones Hirsaugienses et du Summarium Heinrici recoupent celui d'Albert le Grand : «l'esox est un poisson que certains appellent labse, mais d'autres appellent ainsi un grand poisson du Danube et de certains de ses affluents que les Hongrois et Alamans appellent buso. Celui qu'on appelle labse a l'aspect et la couleur du saumon, si ce n'est qu'il a la mâchoire inférieure recourbée vers le haut, comme le bec de l'aigle est recourbé vers le bas, sans être cependant plus longue que celle du haut ; et la mâchoire du haut reçoit celle du bas dans une cavité prévue à cet effet. Sa chair n'est pas aussi rouge ni aussi savoureuse que celle du saumon ${ }^{73}$. " Le mot esox est utilisé par Pline (nat. 9, 44) pour

72 Nous reprenons ici la note que nous avons consacrée à la carpe dans notre édition du Liber de piscibus de l'Hortus sanitatis, Caen, Presses universitaires de Caen, sous presse.

73 Albert le Grand, De animalibus, op. cit., 24, 49 (32) : Esox piscis est quem quidam labsen vocant, quidam autem magnum piscem Danubii et quarundam aquarum Danubium influentium quem Ungari et Alamanni husonem vocant, dicunt esocem. Ille igitur qui labse vocatur figuram et colorem habet salmonis, nisi quod inferiorem mandibulam ad superius habet recurvam sicut rostrum aquilae curvatur ad inferius; et tamen non 
désigner, sans autre précision, un grand poisson du Rhin dans lequel certains auteurs médiévaux ont donc voulu reconnaître l'animal qu'eux-mêmes nommaient labse.

Quelle que soit l'identité du poisson que les Romains connaissaient sous le nom d'esox $x^{74}$, les informations fournies par Albert le Grand (la ressemblance du labse et du salmo, le croc caractéristique du labse et la moindre qualité de sa chair $^{75}$ ) permettent de reconnaître dans salmo et labse le même poisson, le saumon atlantique (Salmo salar Linné, 1758), à des stades différents de son évolution. Lorsque les saumons remontent les cours d'eau, entre juin et novembre, pour atteindre leurs zones de frai, ils se transforment physiquement ; leur peau s'épaissit et change de couleur, les mâchoires inférieures des mâles deviennent crochues; de plus, la remontée des cours d'eau et le frai épuisent la plupart des individus. Lappellation labse désignerait donc le saumon après sa transformation pendant le frai, lorsqu'il a acquis son croc : Gesner ${ }^{76}$ indique ainsi que, sur les rives du Rhin, les termes salmo et lachs désignent le même poisson, salmo étant réservé au saumon pêché du printemps jusqu'au 25 juillet (le saumon de printemps et d'été), lachs au saumon pêché de cette date jusqu'au dernier jour de novembre (le saumon d'automne et d'hiver ${ }^{77}$.

\section{Minuua (Hirsau 24), la chevesne}

Minuua (avec les variantes graphiques muniwa, muniuua, munua) est un nom de poisson d'origine germanique avec un étymon *muniwa (voir nhd Münne). Le terme allemand Münne sert à désigner deux poissons, l'ide mélanote (Leuciscus idus Linné, 1758), un poisson des eaux saumâtres et des cours d'eau, dont

est longior quam superior; sed superior foramine ad hoc parato in se recipit inferiorem. Carnem autem habet non adeo rubeam neque ita delectabilem sicut salmo.

74 De SAInT-Denis, Le vocabulaire des animaux marins..., op. cit., p. XXVII, reste très prudent sur l'identification de l'esox : «Esox, qui désignait un poisson du Rhin (on ne peut préciser davantage : saumon ? esturgeon ? brochet ?), a été accaparé par les naturalistes pour dénommer le genre brochet ». Pour ERnout-Meillet, s.v. esox, esox (isox, isex, issicius) est une désignation du saumon et un mot d'origine étrangère ; de même pour A.C. AndREws, « Greek and Latin Terms for Salmon and Trout », Transactions and Proceedings of the American Philological Association, 1955, vol. 86, p. 308-318. Selon A.C. ANDREWs, le latin aurait connu plusieurs appellations du saumon : esox ou isox, qui aurait été le nom usuel, salmo, d'emploi plus occasionnel, ancoravus ou ancoravo, plus tardif, et enfin tec(c)o, qui nommerait le jeune saumon.

75 Hildegarde de Bingen porte toutefois un jugement contraire à celui d'Albert le Grand et considère que la chair du saumon, molle et faible, ne vaut rien à manger (Physica 5, 5) tandis que celle du lasz serait plus saine, bien qu'elle fasse du mal aux malades (Physica 5, 7).

76 Nomenclator aquatilium animantium, op. cit., p. 327.

77 Voir sur ce point les notes de commentaire de K.F. Kitchell et I.M. Resnick, Albertus Magnus. On animals, op. cit., p. 1682 ; voir aussi L. Moulinier, "La faune germanique médiévale... », op. cit., p. 195. Hildegarde de Bingen distingue elle aussi les poissons salmo et lasz qu'elle traite dans deux notices différentes (salmo en Physica 5, 5 et lasz en Physica 5, 7). Le Summarium Heinrici distingue de même labs et salmo, mais le texte semble à cet endroit perturbé, les deux éditions consultées donnent la même glose gamarus salmo (avec la variante salmo vel poleris) qui s'explique mal, gammarus désignant le homard en latin classique... 
la population a beaucoup diminué de nos jours du fait de la surpêche, mais dont la chair est appréciable et qui peut s'adapter facilement aux viviers; ou bien un poisson qui ressemble à l'ide, mais à la tête large, la chevesne (Leuciscus cephalus Linné, 1758), un poisson des rivières à fort courant ou des lacs, dont la chair, pleine d'arêtes, est de moindre qualité. Le Summarium Heinrici donne la glose capito vel cap ( $p$ ) edo (avec la variante vel dentex) alant (avec la variante alnt) vel munua (avec les variantes munewa, muniva) : or, à côté de Münne, on trouve aussi en allemand les appellations Aland, Alant, Alat pour désigner l'ide ; mais le latin capito (avec la variante capedo), " qui a une grosse tête ", a servi à désigner des poissons à grosse tête dont la chevesne ${ }^{78}$. En l'état actuel des sources disponibles, il est donc difficile de trancher entre deux poissons très proches d'apparence, qui n'étaient peut-être pas différenciés.

Le signe qui sert à nommer le poisson munuиa ne permet guère d'aller plus loin dans son identification, puisqu'il caractérise simplement la munuva comme un poisson de consommation courante (le couteau qu'on aiguise servant à nommer un objet d'utilité quotidienne, et donc un poisson commun).

\section{Ruppa (Hirsau 24), la lote de rivière}

Le mot ruppa doit être rapproché des appellations germaniques de la lote de rivière (Lota lota Linné, 1758) : ahd. ruppa, mhd. rupe, ruppe et, plus tardif, raup ou rutte. Hildegarde de Bingen évoque ainsi l'animal sous le nom d'alroppa (Physica 5, 34), et Albert le Grand propose une synonymie entre le latin médiéval borboth $a^{79}$ et les noms allemands alrutten, alquappen, lumpen, accompagnée d'une description détaillée de l'animal qui permet de reconnaître dans borbotha / alrutten la lote de rivière ${ }^{80}$. Pour nommer la lote d'eau douce, le latin classique disposait du nom mustella, qui a survécu, par exemple dans fr. moutelle, mais qui a été concurrencé par des appellations vernaculaires ; si Albert le Grand est en mesure d'identifier le poisson nommé alrutten avec celui nommé borbotha, en revanche il ne fait pas le rapprochement avec celui que ses sources anciennes appellent mustella. En outre, si l'identification de ruppa semble assurée, en revanche, l'étymologie du mot, parfois rapporté au latin rubeta, "grenouille », demeure incertaine. La lote est un poisson résistant, qui peut être transporté

78 P. Monat, Hildegarde de Bingen, op. cit., p. 108 identifie la monuva décrite par Hildegarde de Bingen (Physica 5, 16) comme le goujon, mais L. Moulinier, "La faune germanique médiévale... ", op. cit., p. 196, considère qu'il s'agit de la chevesne [chevaine]. Chez Vincent de Beauvais, Thomas de Cantimpré, et Albert le Grand le terme capito semble utilisé plutôt pour nommer le chabot que la chevesne.

79 Borbotha est sans doute la latinisation d'une forme vernaculaire à rapprocher de noms de la lote de rivière en ancien français : borbote, bourbote, borbote.

80 Albert le Grand, De animalibus, op. cit., 24, 21 (13) : Borthothae dicuntur pisces fluviales et lacunales anguillis fere similes, sed breviores et magnos ventres habentes, et profunda semper petunt ita quod in lacu Germaniae qui infundit Constantiam ad trecentos passus sub aqua hamis capiantur. Hic piscis Germanice alrutten a quibusdam vel alquappen vocatur, nonnulli etiam lumpen vocant. Carnem habet dulcem, pellem viscosam non spissam, epar magnum et rotundum et dulcissimum. 
et dont la chair comme le foie sont appréciés. Le signe, choisi pour la qualifier, celui du rocher (saxum), peut s'expliquer par le comportement d'un animal nocturne qui reste caché sous les pierres durant le jour.

\section{Sturio (Hirsau 12), l'esturgeon}

Le nom de l'esturgeon sturio / sturgio est un mot d'origine germanique qui remonte à un étymon a. b. frq. *sturjo, de même sens. On le rapprochera des appellations vernaculaires a. h. all. stur(i)o et all. Stör. Le latin disposait du terme ac(c)ipenser pour désigner l'esturgeon, en particulier sous l'espèce ordinaire : Acipenser sturio Linné, $1758^{81}$. Mais alors que les mentions et les descriptions de l'ac(c)ipenser sont nombreuses dans la littérature latine classique et tardive ${ }^{82}$, le mot lui-même n'est pas passé dans les langues romanes (i1 n'aurait survécu que dans quelques dialectes d'Italie du Nord), au contraire du bas latin sturio qui s'est imposé aux dépens de l'ancienne désignation latine ${ }^{83}$. $\mathrm{Si}$, au XVI ${ }^{\mathrm{e}}$ siècle, Gesner établit une synonymie entre acipenser et les noms courants sturio et stora ou les appellations vernaculaires it. sturio, fr. estourgeon, all. Stor, en revanche les auteurs médiévaux ne semblent pas avoir été en mesure de faire le rapprochement entre l'ac(c)ipenser des Anciens et le sturio qui leur était familier : Vincent de Beauvais, comme Thomas de Cantimpré et Albert le Grand consacrent ainsi dans leurs catalogues des animaux aquatiques des notices distinctes à chacune des deux entrées sans suggérer qu'il puisse s'agir du même poisson ${ }^{84}$. Quant au Summarium Heinrici, il donne la glose : rombus sturo, proposant apparemment une équivalence avec l'ancien nom latin du turbot, rhombus, d'autant plus surprenante que les sources anciennes sont assez précises pour identifier le rhumbus au moins comme un poisson plat. La liste d'Hirsau a levé l'ambiguïté de la liste de Cluny en spécialisant dans la désignation du sturio le signe proposé à Cluny pour qualifier soit le saumon soit l'esturgeon et en inventant un signe spécifique pour nommer le saumon. Nous reviendrons sur ces deux signes à propos du saumon.

81 Sur l'identification du poisson accipenser, voir De Saint-Denis, Le vocabulaire des animaux marins..., op. cit., p. 1-3.

82 Ainsi on peut citer Cic. fin. 2, 25 ; 2, 91 ; tusc. 3, 43 ; Hor. sat. 2, 246 ; Mart. 13, 91 ; Ov. hal. 134 ; Plin. nat. 9, 60 ; 32, 145 et 153 ; Ambr. hex. 5, 1, 2 ; Hier. epist. 45, 5 ; et surtout MAcr. sat. 3,16,1, qui consacre un long développement à l'esturgeon.

83 Voir CNRTL s.v. esturgeon ; ERNOUT-MeILLET s.v. acipenser.

84 Vincent de Beauvais, Speculum naturale, op. cit., 17, 64 et 17, 95 ; Albert le Grand, De animalibus, op. cit., 24, 10 (10) et 24, 105 (51), ; THOMAS DE CANTIMPRÉ, Liber de natura rerum, op. cit., 7, 10 et 7,70 . 


\section{B. - Les signes communs aux trois listes}

1. - Deux entrées lucius et salmo sont communes aux trois listes, mais associées à des signes différents. Ces deux noms ont une origine latine.

\section{Lucius (Cluny 13 Fleury 18 Hirsau 16), le brochet}

La plus ancienne attestation du nom lucius est assez tardive puisqu'elle remonte à Ausone (Mos. 120), et on remarquera que Vincent de Beauvais, Albert le Grand et Thomas de Cantimpré ${ }^{85}$ ne traitent du lucius que sous leur propre autorité sans se référer ni citer de sources plus anciennes. En revanche, leurs descriptions répondent assez précisément au comportement et à la morphologie du brochet (Esox lucius Linné, 1758) et témoignent d'une bonne connaissance de l'animal. Le nom latin lucius a des représentants dans les langues romanes anc. fr. lus, luz, it. luccio, ptg. lúcio, esp. Lucio..., et au XVI siècle Gesner ${ }^{86}$ relève encore parmi les noms du lucius usités en France lucz, régionalisme en usage à Bordeaux, à côté des termes brochet, becquet, bechet ou lanzon. Trois des manuscrits des Constitutiones Hirsaugienses glosent lucius par l'appellation vernaculaire hachid (voir all. Hecht ou Heichit, le brochet). De même le Summarium Heinrici pose la synonymie lucius hechit ; et les éditions consultées d'Hildegarde de Bingen donnent la glose lucius pour hecht au chapitre consacré au brochet (Physica 5,9$)^{87}$.

Le nom du lucius est associé par chacune des trois listes à un signe différent. Le geste proposé à Cluny mime la vivacité du brochet qui est, effectivement, capable de bondir sur ses proies en se propulsant d'un coup de queue énergique ; le signe du lucius n'est accompagné d'aucune justification dans la liste d'Hirsau, mais il est probable qu'il fasse allusion au museau plat et large, caractéristique du brochet ; quant au signe de Fleury, qui n’est, lui non plus, développé par aucune explication, il pourrait faire référence à la technique de chasse du brochet, qui s'embusque pour guetter ses proies.

Salmo (Fleury 13, Hirsau 17), le saumon ; salmo vel sturio (Cluny 12), le saumon ou l'esturgeon

85 Vincent de Beauvais, Speculum naturale, op. cit., 17, 29 et 17, 95 ; Albert le Grand, De animalibus, op. cit., 24, 73 (40); THOMAS DE CANTIMPRÉ, Liber de natura rerum, op. cit., 7, 48.

86 Nomenclator aquatilium animantium, op. cit., p. 316.

87 L'étymologie du latin lucius est incertaine; ERnOUT-MeILlet, s.v. lucius, indique que le nom de personne Lucius aurait pu être donné au poisson par dérision (voir Ausone, Mos.), il note que le surnom Lucius rattaché traditionnellement à $l u x$, "la lumière ", aurait pu aussi signifier "le brochet ", comme Gaius, « le geai ». Le nom allemand du brochet. Hecht évoquerait un objet pointu ou crochu, peut-être la dentition redoutable du brochet (voir H. WALTER et P. AvEnAs, La fabuleuse histoire du nom des poissons, Paris, Robert Laffont, 2011, p. 224.). 
L'origine du mot salmo est incertaine, mais le terme est déjà connu de Pline qui l'emploie pour désigner un poisson d'Aquitaine (nat. 9, 68) ${ }^{88}$; toutefois notre enquête ne nous a permis de repérer, outre Pline et surtout Ausone (Mos. 97) qui décrit avec précision le salmo, qu'une seule autre occurrence du mot pour les périodes classique et tardive ${ }^{89}$. L'absence du saumon commun en Méditerranée ou dans les fleuves qui s'y jettent, ou l'existence d'appellations concurrentielles comme esox peuvent expliquer ce constat ${ }^{90}$. En revanche, les mentions du salmo et ses descriptions sont fréquentes chez les auteurs médiévaux, plus familiers des rivières à saumons, et la bonne fortune du terme est attestée par ses représentants dans les langues romanes (fr. saumon, ptg. salmão, esp. salmón, it. salmo, salmone...). Le même signe qui symbolise la richesse et l'orgueil qualifie le saumon dans les listes de Cluny et de Fleury ; la liste de Cluny propose aussi son utilisation lorsqu'il est besoin de nommer l'esturgeon. Il est peu probable qu'il y ait eu confusion de deux poissons aussi physiquement dissemblables que l'esturgeon et le saumon; et, par ailleurs, les sources médiévales montrent qu'il s'agissait alors d'espèces bien identifiées et connues. Il faut plutôt comprendre que les moines de Cluny avaient un usage culinaire comparable de l'esturgeon et du saumon, qui étaient des poissons réputés pour la qualité de leur chair. Les poissons de mer qui remontent les cours d'eau sont particulièrement prisés et recherchés au Moyen Âge. Le saumon en particulier est servi dans les banquets évoqués dans les romans de chevalerie médiévaux : ainsi le graal qu'on apporte au père du Roi pêcheur par antithèse ne contient ni saumon ni lamproie ni brochet, comme on pourrait s'y attendre, mais une seule hostie $^{91}$; quant à l'esturgeon, les auteurs anciens (en particulier Macr. sat. 3, 16), à propos de l'ac(c)ipenser, s'accordent pour voir dans sa chair un aliment de choix; pareillement Albert le Grand précise que le sturio est un poisson réputé dont les chairs sont blanches et savoureuses (De animalibus 24, 105 (51)), et à la fin du Moyen Âge en Angleterre c'est un poisson réservé à la table du roi ${ }^{92}$.

Le catalogue d'Hirsau, plus riche que celui de Cluny et de Fleury, traite séparément du saumon et de l'esturgeon en réservant le signe à double valeur

88 D'après ERnout-Meillet, s.v. salmo, salmo serait un nom d'origine gauloise, qui serait passé en germanique (v.h.al. salmo); sur l'emploi de salmo en latin, voir aussi DE SAINT-DENIS, Le vocabulaire des animaux marins..., op. cit., p. 96-97; ou A.C. AndREWs, « Greek and Latin Terms for Salmon and Trout ", op. cit.

89 Il s'agit du PSEUdo-Gargilius MARTIALIs [fragmenta in codicibus aliquando Medicinis ex oleribus et pomis iuncta, cap. 62] : Capiuntur pisces natura pingues, ut sunt salmones et anguillae et alausae et sardinae vel aringi, et fit ex eis atque ex herbis odoratis aridis cum sale compositio talis.

90 Voir ce point A.C. Andrews, « Greek and Latin Terms for Salmon and Trout », op. cit.

91 Chrétien de Troyes, Conte du Graal, v. 6417-6424 : «Et del riche Pescheor croi / Que il est filz a celui roi / Qui del grail server se fet / Mez ne cuidiez pas que il et / Luz ne lamproie ne saumon : / D'une seule oiste li sainz hon, / Que l'an an cest grail li porte / Sa vie sostient et conforte ».

92 Voir P. MAne, «Images médiévales de la pêche en eau douce ", Journal des savants, 1991, vol. 3, $\mathrm{n}^{\circ} 3-4$, p. 235. On notera qu'à la fin du XVII siècle, le Nouveau commentaire sur l'ordonnance de la Marine du mois d'août 1681, définit encore comme " poissons royaux » tous les poissons qui, par leur rareté ou la délicatesse de leur goût, sont dignes de la table du Roi et y sont ordinairement servis, les dauphins, les esturgeons, les saumons et les truites étant classés comme poissons royaux. 
de Cluny à la seule désignation de l'esturgeon. Il a recouru, pour nommer le saumon, à un signe très proche de celui choisi par Fleury pour qualifier le brochet, entourer l'œil de ses doigts, mais dont nous ne nous expliquons pas la relation avec le saumon.

2. - Les entrées anguilla, lampreda, sepia et truita sont communes aux trois listes et associées aux mêmes signes. Ces noms ont une origine latine plus ou moins ancienne à l'exception de la lampreda.

Anguilla (Cluny 10, Fleury 15, Hirsau 19), l'anguille.

Le terme anguilla est bien attesté depuis le latin classique, par exemple chez Pline, Sénèque, Juvénal, Martial, Macrobe, Isidore de Séville, et il est associé à des descriptions précises qui ne laissent aucun doute sur l'identité de l'animal évoqué, l'anguille (Anguilla anguilla Linné, 1758) ${ }^{93}$. Les auteurs médiévaux s'inscrivent dans cette tradition sans difficulté en reprenant à leur compte ou en complétant les informations qu'ils ont reçues de leurs sources, comme en témoigne la présence, sans solution de continuité, de notices sur l'anguille dans la littérature des bestiaires issue d'Isidore de Séville ${ }^{94}$ ou chez les encyclopédistes du XIII ${ }^{\mathrm{e}}$ siècle ${ }^{95}$; et l'anguille est un des poissons les plus en faveur au Moyen Âge, ce qu'illustre l'épisode du vol des colliers d'anguilles par Goupil dans le Roman de Renart ${ }^{96}$. Le nom latin anguilla a connu une postérité remarquable et il est à l'origine des dénominations courantes de l'animal : fr. anguille, it. anguilla, esp. anguila ${ }^{97}$.

Le signe utilisé pour signifier l'anguille mime un geste de la vie quotidienne (tenir l'anguille des deux mains), mais respecte peut-être plus encore le lien qui associait l'animal à son nom dans une œuvre clé de la culture médiévale, les Étymologies d'Isidore de Séville : «L'anguille tire son nom de sa ressemblance avec le serpent [anguis]. C'est la vase qui la produit ; pour cette raison, elle est si visqueuse que, quand on l'attrape, plus on la serre fort, plus elle glisse rapidement ${ }^{98} »$.

93 Voir, Par exemple, PLin. nat. 9, 74-75 ou 9, 160 ; Isid. orig 12, 6, 41.

94 W.B. Clark, A medieval book of beasts, the second family bestiary, New York, Boydell Press, 2006, p. 210. La recherche des occurrences d'anguilla dans LLT A / B et eMGH montre que le mot a été employé tout au long de la latinité.

95 Thomas de Cantimpré, Liber de natura rerum, op. cit., 7, 2 : cap. De anguilla; Albert le Grand, De animalibus, op. cit., 24, 3 (8) [Anguilla], Vincent DE BEAUvais, Speculum naturale, op. cit., 17, 31 : cap. De anguilla...

96 Voir P. Mane, «Images médiévales de la pêche en eau douce », op. cit., p. 234.

$97 \mathrm{Au}$ début du $\mathrm{XI}^{\mathrm{e}}$ siècle, dans la même aire linguistique et géographique, le Summarium Heinrici glosait le latin anguilla al (avec les variantes aal et ahal, pour al), voir allemand Äal.

98 IsID. orig. 12, 6, 41 : Anguilla dicitur ab anguis similitudine. Hujus origo ex limo est. Unde et, quando capitur, adeo lenis est ut, quanto fortius presseris, tanto citius labatur. Il faut sans doute reconnaître dans la remarque d'Isidore de Séville elle-même un emprunt à la Vulgate de Jérôme : si uelis anguillam aut murenulam strictis tenere manibus, quanto fortius presseris, tanto citius elabitur (HiER., Prologus in libro Iob de hebraeo translato). 
Sepia (Cluny 9, Fleury 14, Hirsau 15), la seiche

Le signe de la sepia est rapporté dans les catalogues de Fleury et de Cluny, mais dans une partie seulement de la tradition textuelle des Constitutiones Hirsaugienses où il ne semble pas avoir figuré à l'origine. Ni le terme sepia, la "seiche » (Sepia officinalis Linné, 1758), ni le signe qui lui est associé, divide I disjunge omnes digitos ab invicem et ita eos commove, ne posent de réelle difficulté d'interprétation. Le mot sepia est attesté sans solution de continuité de l'Antiquité au Moyen Âge et ses emplois n'offrent pas d'ambiguïté quant à l'identification de l'animal désigné sous ce terme ; les auteurs anciens et médiévaux signalent en particulier la capacité de la seiche à émettre une encre noire qui, en troublant l'eau, lui permet d'échapper à ses prédateurs ${ }^{99}$. On retrouve l'étymon latin sepia dans des appellations romanes de l'animal : fr. seiche ${ }^{100}$, it. seppia, catal. cepia, anc. provenç. sepia, ptg. sipia, esp. jibia ou encore dans des termes régionaux. Le latin classique distinguait sans ambiguité, parmi les céphalopodes, deux animaux : la seiche, sepia, et un animal dénommé loligo, qu'on identifie depuis les zoologistes de la Renaissance comme le calmar. On retrouve la distinction sepia / loligo chez les encyclopédistes médiévaux, mais appuyée sur leurs sources livresques antiques plus que sur une expérience effective des réalités zoologiques. On peut alors se demander si, dans le contexte des catalogues des signes, le mot sepia n'avait pas un sens large englobant la seiche et le calmar, les deux étant pêchés le long des rivages européens et étant à la fois comestibles et couramment consommés.

La pharmacopée médiévale, héritière de la médecine gréco-romaine, fait un large usage de l'os de seiche auquel on prêtait des vertus multiples. Vincent de Beauvais consacre ainsi un chapitre complet (Speculum naturale 17, 90) aux remèdes qu'on peut tirer de la seiche, et plus particulièrement de son os ${ }^{101}$. Cependant, les catalogues font apparaître le signe de la seiche dans un contexte sans rapport direct avec la pharmacopée, mais lié à l'alimentation : il faut donc supposer que les moines de Fleury et de Cluny avaient une consommation relativement fréquente de la seiche, un animal marin dont il fallait assurer la conservation et le transport ${ }^{102}$. L'absence du signe relatif à la sepia dans une

99 E. De SAINT-DenIs, Le vocabulaire des animaux marins..., op. cit., p.104. C'est aussi une propriété du calmar.

100 Pour le domaine français, le CNTL fait état d'une forme seche attestée dès le XII siècle, dans Gloss. Tours, L. Delisle (éd.), p. 328 ; en 1197, dans Hélinand DE Froidmont, Les vers de la mort, Fr. Wulff et Em. Walberg (éd.), Paris, Firmin-Didot, 1905, XXX, 11 : saumon et seche. La forme seiche se rencontre au XIII siècle : 1269-78 seiche, dans GUILLAUME DE LORRIS et JEAN DE MEUN, Le roman de la rose, F. Lecoy (éd.), Paris, H. Champion, 1965-1970, 11223.

101 L'os de seiche, utilisé dans la pharmacopée, est large, épais et friable et il se différencie nettement de la «plume » du calmar, fine et presque translucide.

102 Il faut alors supposer avec S.G. BRuce, Silence and Sign Language..., op. cit., p. 81-82, que les moines de Cluny et de Fleury devaient consommer des seiches et des calmars salés et mis en conserve sur leurs lieux de pêche mêmes des côtes méditerranéennes ou atlantiques, puis expédiés à l'intérieur des terres. S.G. BRUCE souligne, à ce propos, que d'autres témoignages confirment l'exploitation de la seiche sur 
partie de la tradition manuscrite du catalogue d'Hirsau est peut-être à expliquer par la situation d'une abbaye éloignée des centres de pêche ou des voies de transport et de commerce de la seiche. Le signe associé à la sepia est une allusion à l'anatomie de la seiche qui possède deux tentacules rétractiles et dont la bouche est entourée d'une couronne de huit bras garnis de ventouses.

\section{Truita, truta (Cluny 14, Fleury 19, Hirsau 18), la truite}

Le nom truita (avec ses variantes tructa / trocta / truta / tritta) désigne communément dans les sources médiévales la truite. Les descriptions du poisson chez les encyclopédistes du XIII ${ }^{\mathrm{e}}$ siècle, par exemple, sont suffisamment précises, qu'il s'agisse de ses caractéristiques morphologiques ou de ses mœurs, pour en permettre une identification certaine, en particulier avec la forme migratrice de la truite de mer (Salmo trutta Linné, 1758). Par ailleurs, les mots issus du latin tructa ont servi à désigner dans les langues romanes la truite : outre fr. truite, on relève aussi it. trota, provenç. trocha, catal. truyta, esp. trucha, ptg. $t r u(i) t a . .$. La signification de tructa est aussi confirmée par les glossaires médiévaux qui posent des équivalences entre le latin tructa et les appellations germaniques de la truite fornha $a^{103}$ : ainsi le Summarium Heinrici 3,16 donne la traduction tactuca (/ lactuca / tactuca) vel trutta (/truita / truta) forhana ${ }^{104}$. Toutefois l'appellation tructa est apparue tardivement en latin, et on note que la truite de mer, que désignent couramment les textes médiévaux sous ce nom, ne se rencontre pas en Méditerranée. Dans la documentation parvenue jusqu'à nous, la plus ancienne attestation du mot remonte à Ambroise où il est donné

\footnotetext{
les rivages de l'Atlantique : ainsi en 1041, Geoffroy de Vendôme et son épouse Agnès assurent à l'abbaye de La Trinité la moitié des revenus qu'ils tirent de l'exploitation des seiches en Saintonge « medietatem quoque nostrae partis de censibus sepiarum per universum pagum Sanctonicum " (Privilegium Gosfredi comitis et Agnetis comitissae, 31 mai 1041). Ce census sepiarum, ce cens perçu sur les seiches, apparaît encore dans une charte antérieure à 1181 citée par LoBINEAU, Histoire de Bretagne, t. 2, col. 137, mentionnée par Du CANGE s.v. census sepiarum: "dederunt medietatem census sepiarum in fluvio Rentia ad S. Ciliacum, excepta Redecima, qua est monachorum S. Martini ». Une enquête sur les droits du roi sur le grau de Salces de 1308 fait aussi mention de cette source de revenus : "debent donationes piscium, census et usatica sepiarum " (É. LALOU, X. HÉLARY. «Enquête sur le grau de Salces (Archives nationales, J 321, n 66) », dans Enquêtes menées sous les derniers capétiens, É. LALOU, Ch. JACOBS (éd.), Paris, Centre de ressources numériques TELMA, 2007). La seiche faisait partie de l'alimentation courante et le Ménagier de Paris, rédigé à la fin

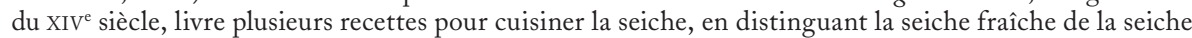
" conrée » (G.E. Brereton, J.M. Ferrier, Le Menagier De Paris. A Critical Edition, Oxford, Clarendon Press, 1981, p. 243). A. DAVRIL, «Le langage par signes chez les moines ", op. cit., p. 72, fait état à ce sujet d'une communication de M.B.H. Bautier, qui lui a indiqué que les seiches arrivaient par tonneaux à Paris aux XIV et XV siècles.

103 C'est le terme retenu par Hildegarde de Bingen, Phys., op. cit., 5, 15 : De Fornha (qu'on rapprochera de l'allemand Forelle).

104 On trouve déjà dans la liste de synonymies latin-germaniques établie par Sedulius Scotus au IX siècle l'équivalence : tructa forahana (De graeca, dans E. vON STEINMEYER / E. Sievers, Die Althochdeutschen Glossen, 2, Glossen zu nichtbiblischen Schriften, Berlin, Weidmannsche Buchhandlung, 1882, p. $623,1.38)$.
} 
comme l'appellation commune d'un poisson nommé varius ${ }^{105}$ sans détail qui permette d'identifier avec certitude le varius. De plus, l'origine de tructa n'est

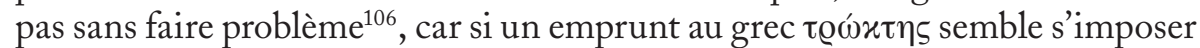
a priori ${ }^{107}$, ce rapprochement pose en fait de réelles difficultés. En effet, les

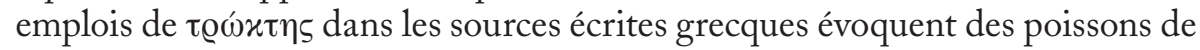
mer qui ont si peu à voir avec la truite qu'on a pu considérer comme fortuite la ressemblance des formes tructa / $\varrho \omega ́ x \tau \eta \varsigma^{108}$ : par exemple, Ernout-Meillet préfère voir dans tructa un mot d'origine gauloise plutôt qu'un emprunt au grec.

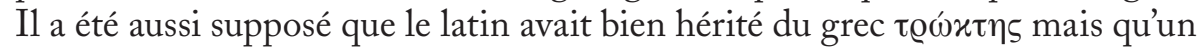
glissement de sens se serait produit une fois le mot passé en latin. Mais, d'un

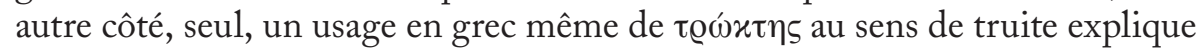
plusieurs désignations vernaculaires de la truite qui remontent à la forme grec-

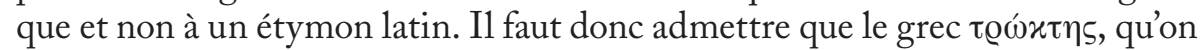
rapproche du verbe $\tau \varrho \omega ́ \gamma \omega$, "dévorer ", a servi dès le monde grec à nommer deux espèces différentes soit la bonite, soit la truite, les deux poissons étant également réputés pour leur voracité, sans qu'on puisse déterminer lequel des

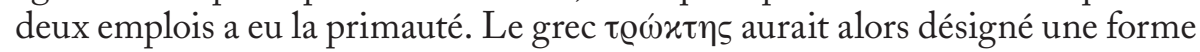
de truite spécifique aux pourtours méditerranéens, la truite à grosses taches (Salmo trutta macrostigma Duméril, 1855). Le latin aurait ainsi disposé d'au moins trois vocables pour désigner les différentes formes de la truite : tructa / trutta, mot d'origine grecque, aurait primitivement désigné soit la truite de lac (Salmo trutta lacustris Linné, 1758) soit la truite à grosses taches ; fario / sario attesté chez Ausone (Mos. 128 sq.), et peut-être à rapprocher du varius, « appelé communément tructa ", d'Ambroise et d'Isidore de Séville, aurait désigné la truite de mer (Salmo trutta Linné 1758), qu'on ne trouve pas en Méditerranée,

105 AmbroIse indique ainsi (hex. 5, 3, 7) : alii ova generant, ut varii maiores quos vocant tructas; on retrouve cette synonymie chez ISIDORE DE SÉviLLE, orig. 12, 6, 6, sans doute reprise à Ambroise : et uarii a uarietate, quos uulgo tructas uocant. On rencontre aussi tructa dans le traité de diététique qu'écrivit au début du $\mathrm{VI}^{\mathrm{e}}$ siècle le médecin Anthimus pour le roi mérovingien Thierry $\mathrm{I}^{\mathrm{er}}$ (Anthimi de observatione ciborum epistula ad Theodoricum regem Francorum, E. Liechtenhan (éd.), Berlin, in aedibus Academiae scientiarum (Corp. med. lat. VIII 1), 1963, 39, p. 18, 1.6 : Tructa et perca aptioris sunt ab aliis piscibus ; dans le Laterculus continens fastos, le calendrier rédigé par Polemius Silvius pour l'évêque Eucher de Lyon vers 448-449 (Polemius Silvius, Laterculus, MGH, auct. ant. IX, chron. min. I, p. 544); chez GrÉGOIRE DE Tours (In gloria martyrum, 75), qui fait état d'une truite d'un poids considérable péché dans le lac de Genève ; ou encore chez SmaragDe, Liber in partibus Donati, ch. De qualitate nominis [CC CM, 68, (B. Löfstedt / L. Holtz / A. Kibre, 1986)], t. 2T, 1. 79 : Piscis species sunt haec : aeses darsus alausa tructa lampreda et reliqua. Smaragde, écolâtre puis abbé de Saint-Mihel sur Meuse, fut actif au début de IX siècle.

106 Nous suivons ici la reconstruction proposée par A.C. ANDREWs, «Greek and Latin Terms for Salmon and Trout », op. cit., p. 308-318, et plus particulièrement, 312-318, qui nous semble la plus précise et la plus convaincante.

107 C'est l'étymologie proposée sans aucune hésitation par J. ANDRÉ dans son édition du livre 12 des Étymologies d'Isidore de Séville, op. cit., p. 185, 333.

108 Ainsi D’Arcy W. Thompson, A Glossary of Greek Fishes, London, Oxford University Press, 1947,

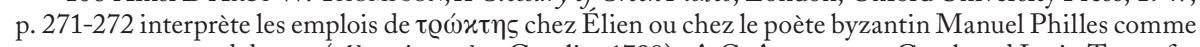
renvoyant au renard de mer (Alopecias vulpes Gmelin, 1789) ; A.C. ANDREWs, «Greek and Latin Terms for

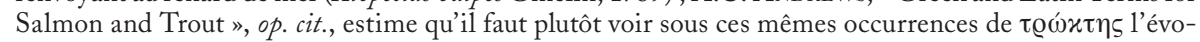
cation d'une bonite, la bonite à dos rayé (Sarda sarda Bloch, 1795) ou la bonite à ventre rayé (Katsuwonus pelamis Linné, 1758). 
mais qui est commune de la Mer Blanche jusqu'aux rivages de l'Espagne ; salar, utilisé par Ausone (Mos. 88 sq.) et par Sidoine Apollinaire (epist. 2, 2), aurait nommé une forme sédentaire, la truite de rivière (Salmo trutta fario Linné, 1758). Le nom tructa serait ensuite devenu un terme générique pour désigner les différentes formes de truite comme en attesteraient les témoignages d'Ambroise et d'Isidore de Séville et la postérité du mot à l'époque médiévale ${ }^{109}$.

Le signe de la truite associe le signe général du poisson et celui de la femme symbolisé par un élément du costume féminin, les ligaturae qui ornent le front des femmes d'un sourcil à l'autre, sans doute le bandeau dont les femmes enserrent le haut du front et recouvrent leur chevelure. Si les listes de Cluny, de Fleury et d'Hirsau n'ont pas d'entrée pour le signe de la femme, en revanche on le retrouve décrit de façon similaire à propos du signe des vierges consacrées : pro signo cuiusque sacre virginis trahe digitum in fronte de supercilio ad supercilium, quod et signum est mulieris, " pour le signe d'une sainte vierge, passe le doigt sur le front d'un sourcil à l'autre, ce qui est aussi le signe de la femme » $(\text { Cluny } 78)^{110}$. On se rappellera que la chevelure retenue par un bandeau ou un voile représente traditionnellement dans la symbolique médiévale la femme mariée ou consacrée tandis que les cheveux dénoués désignent la jeune fille encore vierge ou la femme dissolue ${ }^{111}$. Si on comprend l'allusion générale sur laquelle repose le signe, en revanche, nous n'avons pas retrouvé le sens précis de ces «ligaturas quae in tali loco habentur a feminis ", "de ces ligaturae qu'on trouve en un tel endroit chez les femmes ». Nous n'avons pas relevé, en effet, d'autre occurrence de ligatura pour désigner explicitement un élément du costume féminin présent sur le front entre les sourcils : on rencontre le mot, dans des contextes voisins, pour nommer, soit le fait de retenir les cheveux de façon générale ${ }^{112}$, soit des attaches de coiffe ou de bonnet ${ }^{113}$, soit encore, et fréquemment, des amulettes qu'on suspend pour repousser maladie ou mauvais sort. Nous comprenons donc

\footnotetext{
109 Ce sont les conclusions d'A.C. Andrews, " Greek and Latin Terms for Salmon and Trout », op. cit.

110 On retrouve, avec une légère variante, un geste et une référence vestimentaire comparables dans la liste de Bury St Edmunds éditée par W. JAREcKI, Signa loquendi, op. cit., à propos du signe de Marie : pro signo sancte Marie, fac signum regis premisso signo femine, quod ita fit : tribus digitis ad volam recurvis et pollice extenso latere indicis a timpore in timpus super frontem trahe propter peplum (Bury St Edmunds 47). Le signe associé à la femme semble être une constante dans les listes médiévales si on en juge par les relevés de G. van Rijnberk, Le langage par signes chez les moines, North-Holland publishing Company, Amserdam, 1953, aux entrées femina, p. 62, maria, p. 90, muller [sic] profana, p. 97, virgo, virgo sacra, virgo et martyr, p. 158.
}

111 Sur la symbolique de la chevelure au Moyen Âge, voir en particulier La chevelure dans la littérature et l'art du Moyen Âge: actes du $28^{e}$ colloque du CUER MA, C. Connochie-Bourgne (éd.), Aix-en-Provence, Presses de l'université de Provence, 2004.

112 Par exemple, au XII siècle, dans les Commentaria in Cantica canticorum de PhiLIPPE De Harueng, PL 203, lib. 4, cap. 8, col. 367, 1. 32 : capillos igitur uirginis et bene radicatos reddit quaedam ueluti uis naturae et foris castigatos regularis conuenientia ligaturae cum suos illa sensus subtili prouidentia reddit solidos in affectu et potenti censura dissoluto cohibet ab effectu cum in corde suo sensualibus affectibus modum ponit et foras prodeuntibus disciplinae redimicula mox imponit.

113 Par exemple, au XVI ${ }^{\mathrm{e}}$ siècle, dans Jan van RuUsBroec, Commentaria in tabernaculum foederis, Th. Mertens et G. de Baere (éd.), CC CM, 105-106, 2006, pars 5, cap. 37, 682, 948 : eratque illi 
le mot au sens de bandes, d'attaches (pour maintenir un voile ou pour enserrer les cheveux à la manière d'un bandeau), mais il ne faut peut-être pas exclure un sens, moins probable cependant, de pendeloques. L'explication donnée pour justifier la combinaison du signe de la femme au signe générique du poisson pour caractériser la truite laisse perplexe. Le choix est en effet motivé par des considérations linguistiques, le genre féminin du nom de la truite; or il existe en latin bien d'autres noms de poissons de genre féminin. Peut-être faut-il chercher dans la variante du catalogue d'Hirsau, qui précise que les appellations de la truite sont semper, "toujours ", de genre féminin, le détail qui a retenu l'attention des moines, à savoir que les noms de la truite étaient féminins non seulement en latin, mais dans toutes les langues qu'ils connaissaient. Il est vrai que les monastères rassemblaient alors des individus d'origines linguistiques variées et que la pratique des signes était un des moyens de faire naître ou d'exprimer un sentiment d'appartenance à la communauté ${ }^{114}$.

\section{Lampreda (Cluny 11, Fleury 16, Hirsau 15), la lamproie}

Le mot latin lampreda ne se rencontre pas en latin classique, du moins dans les sources littéraires parvenues jusqu'à nous. Les Romains semblent, en effet, avoir désigné sous le même vocable murena à la fois les murènes si appréciées des riches Romains et les lamproies qu'ils pourraient avoir considérées comme une espèce particulière de murène, si on en juge par le témoignage de Pline (nat. 9, 76), qui mentionne la présence en Gaule septentrionale d'une sorte de murena dont la mâchoire porte une constellation de sept taches dorées : in Gallia septentrionali murenis omnibus dextera in maxilla septenae maculae ad formam septentrionis aureo colore fulgent, dumtaxat uiuentibus, pariterque cum anima extinguuntur, " en Gaule septentrionale, toutes les murènes ont sur la mâchoire droite sept taches disposées comme la grande Ourse qui brillent comme l'or tant qu'elles sont vivantes, mais qui perdent leur éclat à leur mort ». E. De Saint-Denis, à la suite de Cuvier, considère que Pline parle ici « de la lamproie, marbrée comme la murène, mais reconnaissable aux sept ouvertures branchiales qu'elle a de chaque côté de la têt $\mathrm{e}^{115}$ ». Une des attestations les plus anciennes du mot lampreda paraît être le commentaire de Smaragde de Mihel sur l'Ars major de Donat, rédigé vers $825^{116}$. Les emplois de lampreda (on trouve

impositum aliud quoddam velamen byssinum, descendens usque ad imam barbam, celans pilei huius vel Tiarae ligaturas.

114 Sur le rôle du langage des signes dans l'expression d'une appartenance communautaire, voir S.G. BRUCE, Silence and Sign Language..., op. cit., p. 78-79, 90-92.

115 E. De SAInT-Denis, Le vocabulaire des animaux marins..., op. cit., p. 70, d'après G. Cuvier, Le Règne animal distribué d'après son organisation, pour servir de base à l' histoire naturelle des animaux et d'introduction à l'anatomie comparée..., Les Poissons, par A. Valenciennes, Paris, Fortin, Masson et cie, 1843, p. 380-381.

116 La notice étymologique du CNRTL indique un premier emploi de la forme française lamproie en 1178 (Renart, éd. M. Roques, 1971), avec un étymon bas latin lampreda « lamproie » qui serait apparu 
aussi les formes lamprida, lampredo, lamprosis, lamproia et lampetra ${ }^{117}$ relevées par les dictionnaires Du Cange et Blaise ${ }^{118}$ ), comme la postérité de l'étymon latin dans les langues vernaculaires (provenç. lampreza, lamprea, lamprada; catal. lamprea, llamprea; esp. et ptg. lamprea; it. lampreda ; angl. lamprey; all. Lamprete...) ne laissent subsister aucun doute sur l'identification du poisson avec la lamproie. Les trois espèces de lamproies qu'on peut trouver en Europe, où elles sont abondantes, sont la lamproie de rivière (Lampetra fluviatilis Linné, 1758), la lamproie marine (Petromyzon marinus Linné, 1758) et la lamproie de Planer, les deux premières étant des espèces migratrices qui reviennent dans les eaux douces pour s'y reproduire. Les lamproies peuvent aisément êtres distinguées des autres poissons par leur disque buccal et leurs sept paires de fentes branchiales.

La synonymie précisée dans les catalogues de Fleury et d'Hirsau, murena vel / sive lampreda / lamprida est attestée couramment au Moyen Âge. C'est ainsi, par exemple, que le Summarium Heinrici introduit la désignation vernaculaire de la lamproie : ysmerena grece, murena latine vel lampreda lamphrida (avec, pour lamphrida, les variantes lantfrida / lampfrid/lamfrida / lamprida /lanfrida). Certes la cavité buccale des deux animaux est bien différente : la murène est

au VIII ${ }^{\mathrm{e}}$ siècle ; notre enquête dans la base Brepolis (recherches croisées sur la Library of Latin texts, séries A et B et sur les Monumenta Germaniae Historica) nous a fourni comme plus ancienne attestation le Liber in partibus Donati de Smaragde, ch. De qualitate nominis, op. cit. : Piscis species sunt haec : aeses, darsus, alausa, tructa, lampreda et reliqua.

117 L'origine du latin lampreda est incertaine et nous avouons notre perplexité devant l'hypothèse la plus couramment avancée qui explique lampetra à partir de lambere petras, « lécher les pierres ». Cette interprétation que mentionne, en la critiquant, le dictionnaire Du CANGE, s.v. lampetra, semble remonter à Niccolò Perotti (Nicolaus Perottus 1429/1430-1480) : mustellae [...] quas a lambendis petris uulgo nunc lampetras nominant (Cornucopiae siue Commentarii linguae latinae... que nous avons consulté dans l'édition de Bâle, Cratander, 1521, p. 159, 1.18-20). C'est une étymologie qui a été largement diffusée par les glossaires et les lexiques à partir du XVI siècle (ainsi A. Furetière, Dictionaire [sic] Universel, La Haye et Rotterdam, Arnout et Reinier Leers, 3 tomes, 1690 ; édition électronique critique par Cl, Classiques Garnier Numérique, 2003, s.v. lamproye, et elle a sans doute été inspirée à Niccolò Perotti par le disque buccal de la lamproie qui fait ventouse et lui permet de se fixer à d'autres poissons ou à des pierres. Peut-être a-t-elle influencé Linné lui-même dans sa dénomination du genre des lamproies Petromyzon, littéralement " suce-pierre » ou « lèche-pierre », néologisme qu'il a inventé à partir du grec petros, "pierre ", et myzein, "lécher ». A. REY (s.v. lamproie), à propos de cette étymologie traditionnelle qui fait venir lampreda de lampetra, note qu'une reconstruction inverse est tout autant plausible : un rapprochement étymologique douteux avec lambere et petra ayant pu entraîner la corruption d'une forme initiale lampreda en lampetra. Plus récemment (voir notice étymologique « lamproie » du CNRTL ; voir aussi C. WILLE, « Der Reiher, das Neunauge und der Igel. Tiernamen im romanischen Mittelalter ", dans Tiere Und Fabelwesen Im Mittelalter, S. Obermaier (éd.), de Gruyter, Berlin, 2009, p. 93-95), on a pu rapprocher lampreda d'une autre désignation de la lamproie en latin tardif, naupreda, attestée plus anciennement que lampreda, avec une occurrence dans le Laterculus continens fastos, de PolEmius Silvius, op. cit., I, p. 544). On trouve aussi les leçons naupreda et nauprida à côté de lampreda dans certains manuscrits du De observatione ciborum epistula d'Anthimus, op. cit., 47, p. 19, 1. 14. H. Walter et P. Avenas, La fabuleuse histoire du nom des poissons, Paris, Robert Laffont, 2011, p. 102, se font l'écho de cette hypothèse et considèrent lampreda comme une déformation de naupreda sous l'influence de lambere, "lécher ». Ils voient dans naupreda la combinaison de navis, «navire » et de praeda, «proie » et expliquent cette étymologie par le fait que les lamproies peuvent s'attacher à la coque des bateaux. Toutefois cette explication ne fait pas l'unanimité, et l'origine de naupreda / nauprida reste encore discutée.

118 Consulté sur Brepolis, DLD. 
dotée d'une mâchoire aux dents redoutables, tandis que la lamproie n'a pas de mâchoire, mais un disque buccal. Cependant il faut reconnaître que la murène et la lamproie sont deux poissons longs, marbrés et anguilliformes dont la peau est dépourvue d'écailles et recouverte de mucus ; de plus, la lamproie est au Moyen Âge un mets recherché comme l'était la murène à Rome, ce qui a pu favoriser la synonymie des deux termes, sinon la confusion entre les deux animaux $^{119}$. L'invention du signe qui décrit la lamproie dans le lexique des signes monastiques a sans doute été motivée par la présence des sept paires de pores branchiaux de la lamproie.

Ainsi, les noms et les signes utilisés pour désigner les poissons dans les catalogues de Cluny, Fleury et Hirsau témoignent que les moines qui les ont composés se sont appuyés sur une connaissance plus empirique que livresque. En effet, on constate, d'une part, que de nombreux noms de poisson sont d'attestation tardive ou d'origine germanique, et, d'autre part, que le signe utilisé correspond largement soit à un détail physique, soit à un comportement particulier de l'animal facilement observable par celui qui le cuisine ou le pêche. Ainsi, parmi les dix-huit noms de poissons retenus, seuls l'allec, l'anguilla, le salmo, la sepia et le cancer sont attestés à date ancienne ; en outre, parmi ces derniers, l'allec a pris un sens différent de celui qu'il connaissait à l'époque antique, et il n'est pas sûr que salmo, qui apparaît une seule fois chez Pline, ait désigné chez lui le saumon. Il faut citer aussi le nom murena, mais il est probable que celui de lampreda ait été plus fréquemment employé, puisque murena est absent du catalogue de Cluny, tandis que Fleury et Hirsau le mentionnent pour souligner son équivalence avec lampreda. Ce dernier est absent des textes anciens, mais les formes dans les différentes langues vernaculaires indiquent qu'il est d'origine latine. Lucius et truita / truta sont inconnus avant Ausone ou Ambroise. Enfin, si le vernaculaire barbo vient du latin barbus, il n'est pas sûr que la forme du catalogue ne soit pas un calque de l'allemand, tandis que le terme muletus, comme nom de poisson, est sans doute une réfection de l'ancien français mul(1)et. Quant aux autres noms de poissons, asco, brashima, carpho, lasho, minuua, ruppa, sturio, que l'on ne trouve qu'à Hirsau, ils ont tous une origine germanique et témoignent ainsi pour certains de la préférence accordée au nom d'origine vernaculaire, c'est-à-dire à la transmission orale.

119 Sur la faveur dont jouissait la murène auprès des riches romains, voir E. DE SAINT-Denis, Le vocabulaire des animaux marins..., op. cit., p. 69-71; sur la place de la lamproie dans la culture médiévale, voir C. Wille, «Murena id est Lampreda: Quelques observations lexicologiques et culinaires », Reinardus: Yearbook of the International Reynard Society 20, 2007-2008, p. 170-187. Pierre Damien rappelle ainsi les exemples édifiants d'un abbé et d'un évêque qui manquent de perdre leur âme pour de savoureuses lamproies... Petrus Damiani, Epistulae, K. Reindel (ed.), MGH, Epistolae, Die Briefe der deutschen Kaiserzeit 4, 2 Die Briefe des Petrus Damiani 41-90, p. 176-177 [Epist. 57]. 
De même, le choix des signes est motivé, comme nous l'avons dit, par une caractéristique du poisson, le plus souvent physique ou comportementale. I1 fallait individualiser un trait de l'animal facile à mémoriser, donc évident et bien connu des moines, et l'associer avec un signe qui ne présente pas de difficulté dans sa réalisation et aisément reconnaissable. On ne s'étonne donc pas du signe qui désigne, d'une manière qui nous semble encore aujourd'hui tout à fait bien choisie, le hareng à Hirsau, l'anguille, le barbeau, le « crabe », la lamproie, le brochet à Cluny, le mulet ou la seiche. D'autres signes reposaient sur une observation plus fine du poisson comme celui de l'ombre - remarquable par ses écailles - ou de la carpe ; d'autres encore impliquaient, semble-t-il, une connaissance des mœurs de l'animal, comme celui de la lote ; en revanche, le signe de l'esturgeon et du saumon peut venir d'une tradition autant livresque qu'orale, qui nous échappe en partie, de même que l'interprétation d'un certain nombre de signes pour lesquels on en est réduit à formuler des hypothèses. Mais il n'en demeure pas moins que ces catalogues constituent une source utile à l'étude du savoir sur les poissons au Moyen Âge et complémentaire du savoir transmis par les savants de langue latine depuis Isidore de Séville : il témoigne notamment, grâce à la perméabilité du latin aux langues vernaculaires, d'un vocable inconnu de ces derniers, mais dont l'emploi était assez courant pour un certain nombre d'entre eux pour se maintenir dans les langues d'aujourd'hui. 


\section{Annexe}

Liste des poissons selon leur ordre d'apparition dans les catalogues de Cluny, Fleury et Hirsau

\begin{tabular}{|c|c|c|c|}
\hline \multirow{2}{*}{$\begin{array}{l}\text { Cluny } \\
\text { W. Jarecki (éd.), } \\
1981\end{array}$} & \multirow{2}{*}{$\begin{array}{l}\text { Fleury } \\
\text { W. Jarecki (éd.), } 1981\end{array}$} & \multicolumn{2}{|l|}{ Hirsau } \\
\hline & & W. Jarecki (éd.), 1981 & P. Engelbert (éd.), 2010 \\
\hline 8 piscis & 12. pisces & 11. piscis & piscis \\
\hline $\begin{array}{l}9 \text { sepiae } \\
\text { les seiches }\end{array}$ & $\begin{array}{l}\text { 13. alec } \\
\text { le hareng }\end{array}$ & $\begin{array}{l}\text { 12. sturio } \\
\text { l'esturgeon }\end{array}$ & \begin{tabular}{|l} 
sturio \\
l'esturgeon \\
\end{tabular} \\
\hline $\begin{array}{l}10 \text { anguilla } \\
\text { l'anguille }\end{array}$ & $\begin{array}{l}\text { 14. sepiae } \\
\text { les seiches }\end{array}$ & $\begin{array}{l}\text { 13. salmo } \\
\text { le saumon }\end{array}$ & $\begin{array}{l}\text { salmo } \\
\text { le saumon }\end{array}$ \\
\hline $\begin{array}{l}11 \text { lampreda } \\
\text { la lamproie }\end{array}$ & $\begin{array}{l}\text { 15. anguilla } \\
\text { l'anguille }\end{array}$ & $\begin{array}{l}\text { 14. lahso } \\
\text { le saumon }\end{array}$ & $\begin{array}{l}\text { lahso } \\
\text { le saumon }\end{array}$ \\
\hline $\begin{array}{l}12 \text { salmo vel sturio } \\
\text { le saumon ou l'esturgeon }\end{array}$ & $\begin{array}{l}\text { 16. murena vel lamprida } \\
\text { la murène ou la lamproie }\end{array}$ & $\begin{array}{l}\text { 15. murena sive lampreda } \\
\text { la murène ou la lamproie }\end{array}$ & $\begin{array}{l}\text { murena siue lampreda } \\
\text { la murène ou la lamproie }\end{array}$ \\
\hline $\begin{array}{l}13 \text { lucius } \\
\text { le brochet }\end{array}$ & $\begin{array}{l}\text { 17. salmo } \\
\text { le saumon }\end{array}$ & $\begin{array}{l}\text { 16. lucius }{ }^{120} \\
\text { le brochet }\end{array}$ & $\begin{array}{l}\text { sepiae } \\
\text { les seiches }\end{array}$ \\
\hline \multirow[t]{13}{*}{$\begin{array}{l}14 \text { truita } \\
\text { la truite }\end{array}$} & $\begin{array}{l}\text { 18. lucius } \\
\text { le brochet }\end{array}$ & $\begin{array}{l}\text { 17. carpho } \\
\text { la carpe }\end{array}$ & $\begin{array}{l}\text { lucius } \\
\text { le brochet }\end{array}$ \\
\hline & $\begin{array}{l}\text { 19. truita } \\
\text { la truite }\end{array}$ & $\begin{array}{l}\text { 18. truitta } \\
\text { la truite }\end{array}$ & $\begin{array}{l}\text { carpho } \\
\text { la carpe }\end{array}$ \\
\hline & $\begin{array}{l}\text { 20. muletus } \\
\text { le mulet }\end{array}$ & $\begin{array}{l}\text { 19. anguilla } \\
\text { l'anguille }\end{array}$ & $\begin{array}{l}\text { truita } \\
\text { la truite }\end{array}$ \\
\hline & & $\begin{array}{l}\text { 20. barbo } \\
\text { le barbeau }\end{array}$ & $\begin{array}{l}\text { anguilla } \\
\text { l'anguille }\end{array}$ \\
\hline & & $\begin{array}{l}\text { 21. asco } \\
\text { l'ombre }\end{array}$ & $\begin{array}{l}\text { barbo } \\
\text { le barbeau }\end{array}$ \\
\hline & & $\begin{array}{l}\text { 22. allec } \\
\text { le hareng }\end{array}$ & $\begin{array}{l}\text { asco } \\
\text { l'ombre }\end{array}$ \\
\hline & & $\begin{array}{l}\text { 23. brahsima } \\
\text { la brème }\end{array}$ & $\begin{array}{l}\text { allec } \\
\text { le hareng }\end{array}$ \\
\hline & & $\begin{array}{l}\text { 24. ruppa } \\
\text { la lote }\end{array}$ & $\begin{array}{l}\text { brahsima } \\
\text { la brème }\end{array}$ \\
\hline & & $\begin{array}{l}\text { 25. piscis assus } \\
\text { le poisson grille }\end{array}$ & $\begin{array}{l}\text { ruppa } \\
\text { la lote }\end{array}$ \\
\hline & & $\begin{array}{l}\text { 26. cancer } \\
\text { «le crabe» }\end{array}$ & $\begin{array}{l}\text { minuua } \\
\text { la chervesne? }\end{array}$ \\
\hline & & & $\begin{array}{l}\text { pisces minuti } \\
\text { les très petits poissons }\end{array}$ \\
\hline & & & $\begin{array}{l}\text { piscis assus } \\
\text { le poisson grillé }\end{array}$ \\
\hline & & & $\begin{array}{l}\text { cancer } \\
\text { «le crabe» }\end{array}$ \\
\hline
\end{tabular}

120 Le signe des sepiae est rejeté comme variante dans l'apparat critique.

121 Les signes de la minuиa et des pisces minuti sont rejetés dans l'apparat critique comme variantes. 


\section{Résumé}

Des poissons, des mots et des signes : les signes monastiques des noms de poissons au XI ${ }^{\mathrm{e}}$ siècle. - Les catalogues des signes, composés dans les abbayes de Cluny, Fleury et Hirsau à la fin du XI siècle, devaient permettre aux moines de communiquer sans enfreindre la règle du silence. Comme cette règle s'appliquait notamment dans la cuisine et le réfectoire, ces catalogues donnent des informations utiles sur les pratiques alimentaires, et notamment sur les poissons, dont on ne trouve pas moins de 18 noms dans le catalogue d'Hirsau. Dans cet article, on se propose d'examiner dans quelle mesure les catalogues peuvent constituer des sources du savoir ichtyologique, grâce au lien instauré entre le nom et le geste. Dans un première partie, on propose une traduction française des signes des poissons, en tenant compte des variantes entre les trois catalogues, et ainsi une identification de chaque animal; puis, dans une seconde partie, après avoir dégagé les critères généraux qui ont guidé le choix des signes servant à figurer les poissons, on justifie l'identification donnée à chacun d'eux, par l'analyse du nom lui-même et du geste qui lui est associé.

Mots-clés : signes monastiques - catalogue des signes - Cluny - Hirsau - Fleury poisson(s) - savoir ichtyologique - vocabulaire latin. 